\title{
Derivation of a New Equation to Obtain the Permeability Coefficient of Clayey Soils
}

\author{
Maysm Th. Al-Hadidy ${ }^{1}$
}

\begin{abstract}
The main objective of this research is to find the coefficient of permeability (k) of the soil and especially clayey soil by finding the degree of consolidation (rate of consolidation). New modify procedure is proposed by using the odometer (consolidation) device. The ordinary conventional permeability test usually takes a long time by preparing and by testing and this could cause some problems especially if there is a need to do a large number of this test and there were a limited number of technicians and/or apparatus. From this point of view the importance of this research is clear, since the modified procedure will require a time of 25 minute only.

Derivation made to produce an equation which could be used to fined the permeability if the proposed procedure fallowed to find the permeability of soils and this done by specification the degree of consolidation at any loading stage.

The results of permeability found by the proposed procedure and by ordinary test (directly by falling head method, and indirectly by accelerated consolidation method using the oedometer device). After that these results were found by proposed procedure compared with that results which found by ordinary test. it has been found that this equation give a very good results with (95.83) \% accuracy and degree of correlation of $(\mathbf{0 . 9 9 8 8 )}$ comparing with ordinary methods and beside that it takes a very short time.
\end{abstract}

Keywords:

coefficient of permeability, compressibility of clay, permeability test, conventional consolidation test, saturated clay, falling head method

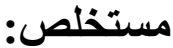

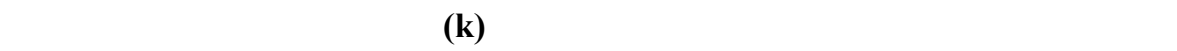

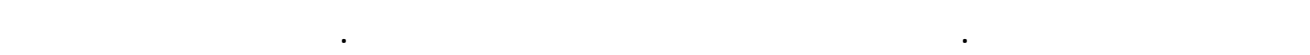

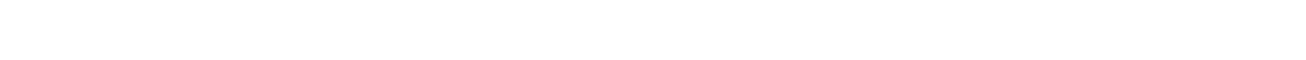

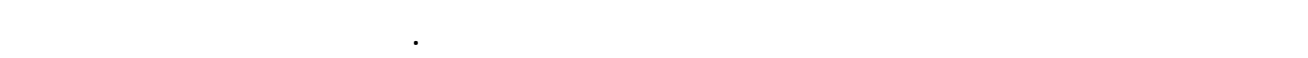

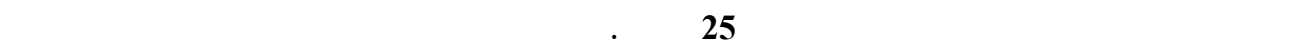

${ }^{1}$ Lecturer, University of Baghdad, College of Engineering, Civil Eng. Dept. 


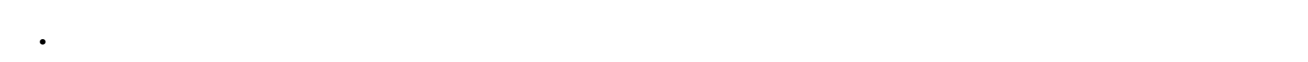

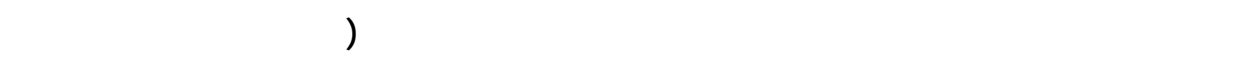





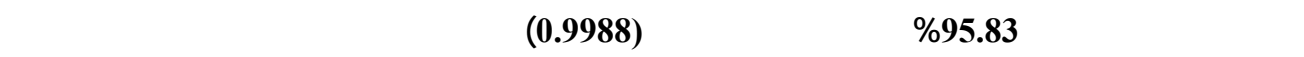

\section{Introduction}

تحتاج إلى قوت قصير.

The knowledge of the permeability of soils, as expressed by the coefficient $k$, is of considerable importance for many practical engineering problems, such as the water retaining capacity of earth dams, the capacity of pumping installations for the lowering of the ground-water level during excavations, and the rate of settlement of building. (Gregory, 1955).

\section{Permeameters:}

The law for flow through soils is named after Darcy in (1856) who demonstrated experimentally that the rate of flow is proportional to the gradient. Darcy's low is written

$$
\begin{aligned}
& Q=k i A \\
& \text { or } \\
& \frac{Q}{A}=v=k i
\end{aligned}
$$

where:

$$
\begin{aligned}
& k=\text { coefficient of permeability. } \\
& i=\text { hydraulic gradient } h / L \\
& L=\text { flow path } \\
& h=\text { hydraulic head } \\
& Q=\text { The quantity of flow } \\
& \text { A }=\text { a cross section of area }
\end{aligned}
$$

$v=$ discharge velocity, which is due quantity of water flowing in unit time through a unit gross cross section area of soil at right angle to the direction of flow. 
The various types of apparatus which are used in soil laboratories for determining coefficients of permeability of soils are called permeameters. They are of two basic designs, the constant head type and the variable head type. (Taylor, 1963)

The constant head permeameter used for permeability down to about $\left(10^{-2} \mathrm{~cm} / \mathrm{s}\right)$, and the falling head permeameter used for values of $k$ between $\left(10^{-2}\right.$ and $\left.10^{-5} \mathrm{~cm} / \mathrm{s}\right)$.

Below about $10^{-5} \mathrm{~cm} / \mathrm{s}$ permeability can generally only be measured by indirect means, in a consolidation test. (Scott, 1980)

\section{Previews Empirical and theoretical relations:}

Hazen's approximation:

Hazen suggested the following empirical relation for the permeability of filter sands

$$
k=C \times\left(D_{10}\right)^{2} \quad m / s
$$

Where

$$
\begin{aligned}
& k=\text { coefficient of permeability. } \\
& D_{10}=\text { the effective size in mm }
\end{aligned}
$$

$C=$ a coefficient varying between 0.01 and 0.015

The approximation is reasonably good for the type of material for which it was proposed, but the expression makes no allowance for variation in porosity, no in the shape of the particles. (Scott, 1980)

\subsection{The Kozeny-Carman equation:}

It may be shown theoretically that, for laminar flow in saturated soils,

$$
k=\frac{1}{k_{o} k_{T} S_{s}{ }^{2}} \cdot \frac{n^{3}}{(1-n)^{2}} \cdot \frac{\gamma_{w}}{\mu}
$$

Where

$K$ is coefficient of permeability.

$k_{o}$ is a factor depending on the shape of the pores. 
$k_{T}$ is a factor depending on the 'tortuosity' of the pores (that is , no the mean distance traveled by the water in moving between two points which are unit distance apart in a straight line).

$S_{s}$ is the surface area of the particles in unit volume of the solid material.

$\gamma_{w}$ is the unit weight of permeant

$\mu=$ viscosity of permeant

$n=$ porosity

For coarse grained soils $k_{o} k_{T}$ is about 5.0, so that

$$
k \cong \frac{1}{5.0 S_{s}^{2}} \cdot \frac{n^{3}}{(1-n)^{2}} \cdot \frac{\gamma_{w}}{\mu}
$$

Kozeny-Carman equation is reasonably reliable in predicting the permeability of coarse-grained soils, and it is not so useful in the case of silts and clays. (Carman, 1956)

\section{The Terzaghi Consolidation Theory:}

The theoretical study of consolidation has for its purpose the setting up of an equation from which the pressure and void ratio values may be known at any point and at any time in a stratum of consolidating soil of any thickness. From such an equation the change in overall thickness of the strata after any interval of time may readily be determined by integration. (Taylor, 1963).

\section{The derivation of proposed equation:}

$$
\begin{aligned}
& k=C_{v} \times m_{v} \times \gamma_{w} \\
& m_{v}=\frac{a_{v}}{1+e_{o}} \\
& \Delta e=e_{0}-e_{i} \\
& a_{v}=\frac{\Delta e}{\Delta p}
\end{aligned}
$$

Where:

$e_{0}=$ initial void ratio. 
$e_{i}=$ the void ratio at the load increment

$\Delta e=$ corresponding change in void ratio.

$\Delta p=$ change in applied stress

$k=$ coefficient of permeability.

$m_{v}=$ coefficient of volume change.

$a_{v}=$ coefficient of compressibility

$C_{v}=$ coefficient of consolidation.

$\gamma_{w}=$ unit weight of water in $\mathrm{kn} / \mathrm{cm}^{3}$

$\varepsilon_{v}=\varepsilon_{h}+2 \varepsilon_{r}$

Where:

$\varepsilon_{v}=$ volumetric strain.

$\varepsilon_{h}=$ axial strain.

$\varepsilon_{r}=$ Radial strain

In consolidation theory $\varepsilon_{r}=0$

Then $\varepsilon_{v}=\varepsilon_{h}$

equation (11) Found from equation (10-b)

$$
\frac{\Delta H}{H}=\frac{\Delta e}{1+e_{o}}
$$

By substituted equation (11) in equation (8) the following equation (12) found

$$
a_{v}=\frac{\Delta H}{H \Delta p} \times\left(1+e_{o}\right)
$$

And by substitute equation (12) in equation (7), equation (13) Found as bellow:

$$
m_{v}=\frac{\Delta H}{H} \times \frac{1}{\Delta P}
$$

This could be written as below in equation (14)

$$
m_{v}=\frac{\text { dial factor }\left(R_{O(p=0)}-R_{O(P=i-1)}\right)}{H_{\text {final }(p=i-1)}} \times \frac{1}{\Delta P}
$$


Where

$\Delta H=\Delta R \times$ dial factor.

$C_{V}=\frac{T\left(H_{a v .}\right)^{2}}{t_{T}}$

where

$C_{v}=$ coefficient of consolidation.

$T=$ time factor

$t_{T}=$ time for the corresponding time factor $T$

$H_{a v}$ = average height of sample

The consolidation ratio defined in (Holtz and Kovacs, 1981) as:

$U_{Z}=\frac{e_{0}-e_{1}}{e_{0}-e_{2}}$

Where:

$e_{1}$ In some intermediate void ratio, as shown on figure (1) what we are looking at graphically in that figure is the ratio of ordinates corresponding to $\mathrm{AB}$ and $\mathrm{AC}$. 


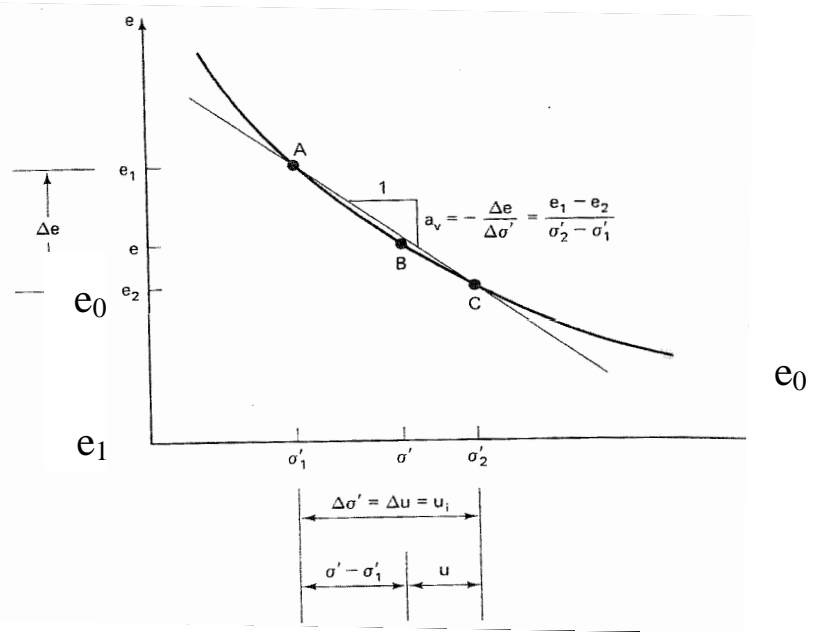

Figure (1): Laboratory Compression Curve. After (Holtz and Kovacs, 1981)

If $e_{0}-e_{1}$ is the different void ratio for proposed period and $e_{0}-e_{2}$ is the different void ratio along the loading stage then: $\left(e_{1}-e_{o}\right) \cdots$ related with $\Delta H_{t(P=i)}$

and $e_{0}-e_{2} \cdots$ related with $\Delta H_{\text {final }(P=i)}$

Then equation 8 could be writing for $\Delta H_{t(P=i)}$ and $\Delta H_{\text {final }(P=i)}$ as below: $\Delta H_{t(P=i)}=\frac{e_{0}-e_{1}}{1+e_{0}} H_{0(P=i)}$ And $\Delta H_{\text {final }(P=i)}=\frac{e_{0}-e_{2}}{1+e_{0}} H_{0(P=i)}$ Then equation (16) writes as below in equation (17)

$$
U_{Z}=\frac{\Delta H_{t(P=i)}}{\Delta H_{\text {final }(P=i)}}=\frac{H_{O(P=i)}-H_{t(P=i)}}{H_{O(P=i)}-H_{\text {final }(P=i)}}=\frac{R_{O(P=i)}-R_{t(P=i)}}{R_{O(P=i)}-R_{\text {final }(P=i)}}
$$

Where:

$H_{O(P=i)}=$ initial height of the sample at that loading stage. 
$H_{t(P=i)}=$ height of sample at the proposed reading time.

$H_{\text {final }(P=i)}=$ height of sample at the end of this loading stage.

$R_{O(P=i)}=$ dial gage reading at the start of that loading stage.

$R_{t(P=i)}=$ dial gage reading at the proposed reading time of that loading stage.

$R_{\text {final }(P=i)}=$ dial gage reading at the end of this loading stage.

And by substitute equations (14 and 15) in equation (6) the coefficient of permeability defined as shown in equation (18)

$$
\begin{aligned}
& k=\frac{T\left(H_{a v .}\right)^{2}}{t_{T}} \times \frac{(\Delta R \times \text { dial factor })}{H_{\text {final }(P=i-1)}} \times \frac{1}{\Delta P} \times \gamma_{w} \\
& \text { If } H_{a v .}=H_{\text {final }(P=i-1)}-\frac{\left(H_{0(P=i)}+H_{t(P=i)}\right)}{2}
\end{aligned}
$$

after bowels (1981)

Then equation (18) became:

$$
k=\frac{T\left(H_{\text {final } p=-i-1)}-\frac{\text { dialfacto }\left(r R_{o(p=i)}-R_{t(P-i)}\right)}{2}\right)^{2}}{t_{T}} \times \frac{\text { dialfactor }\left(r R_{t(P=i)}-R_{o(P-i)}\right.}{H_{\text {final } P=-i-1)}} \times \frac{1}{\Delta P} \times \gamma_{w}
$$

If dial factor $=0.002 \mathrm{~mm} / \mathrm{div}$.

And $t_{\mathrm{T}}$ in min and $\mathrm{H}$ in $\mathrm{cm}$ and two way dranage then:

$$
H_{\text {final }(p=i-1)}=H_{O(p=0)}-0.0002\left(R_{0(p=0)}-R_{\text {final }(P=i-1)}\right)
$$

where

$\mathrm{H}_{0(\mathrm{p}=0)}=$ initial height of the sample at applied load $=0 \mathrm{kpa}$

$R_{0(p=0)}=$ dial reading at the start of testing, time $=0$ min at applied load $=0$ $\mathrm{kpa}$

$R_{\text {final( } P=i-1)}=$ dial reading at the end of previous loading stage.

$T=$ time factor 
$t_{T}=$ time for the corresponding time factor $T$

Then equation 20 became:

$$
\begin{aligned}
k= & \left.\frac{T}{120000001 P t_{T}}\left[H_{O(p=0)}-0.0002\left(R_{O(p=0)}-R_{\text {final }\left(P_{i-1}\right)}\right)-0.0001 R_{0(p=i)}-R_{t(p=i)}\right)\right]^{2} \\
& \times\left[\frac{\left(R_{O(p=i)}-R_{t(p=i)}\right)}{H_{O(p=0)}-0.0002\left(R_{0(p=0)}-R_{\text {final }([-i-1)}\right)}\right]
\end{aligned}
$$

Where:

$$
T=1.781-0.933 \log \left(100\left(1-\frac{\left(R_{O(P=i)}-R_{t(P=i)}\right.}{\left(R_{O(P=i)}-R_{f i n a l(P=i)}\right.}\right)\right) \quad \text { If } \mathrm{U}_{\mathrm{Z}}>60 \%
$$

Or $T=\frac{\pi}{4} \times\left(\frac{\left(R_{O(P=i)}-R_{t(P=i)}\right.}{\left(R_{0(P=i)}-R_{\text {final }(P=i)}\right.}\right)^{2} \quad$ If $\mathrm{U}_{\mathrm{Z}}<60 \%$

\section{The experimental work:}

The experiments were carried out on soil specimens from 17 different locations in Iraq with 54 samples fallowing the sequence below: Each sample from the 54 sample tested by two series of tests Series ( A) where Tests were carried out on soil samples with ordinary permeability test by using the variably head method for clayey soils. And series (B) where modified test were carried out on soils samples by using the Consolidation device by using soil samples $50 \mathrm{~mm}$ diameter at near equal bulk densities.

The general soil properties such as soil texture, density, water content and specific gravity of the soils are given in Table (1).

\section{Results and Discussion:}

By fallowing the experimental program the following results data shown in table (2) for all the samples: 
Table (1) soil samples properties

\begin{tabular}{|c|c|c|c|c|c|}
\hline $\begin{array}{l}\text { Sample } \\
\text { no. }\end{array}$ & location & $\begin{array}{c}\text { unit } \\
\text { weight } \\
\mathrm{kn} / \mathrm{m}^{3}\end{array}$ & $\begin{array}{l}\text { specific } \\
\text { gravity }\end{array}$ & $\begin{array}{c}\text { water } \\
\text { content } \%\end{array}$ & soil description \\
\hline 1 & $\begin{array}{l}\text { baghdad -arab } \\
\text { jbour }\end{array}$ & 19.32 & 2.78 & 22.71 & $\begin{array}{c}\text { brown silty clay stiff to } \\
\text { medium }\end{array}$ \\
\hline 2 & $\begin{array}{l}\text { baghdad -arab } \\
\text { jbour }\end{array}$ & 18.68 & 2.79 & 30.13 & $\begin{array}{l}\text { gray to brown silty clay to } \\
\text { clayey silt medium to stiff }\end{array}$ \\
\hline 3 & $\begin{array}{l}\text { baghdad -arab } \\
\text { jbour } \\
\end{array}$ & 20.42 & 2.8 & 23.57 & $\begin{array}{c}\text { brown silty clay very stiff with } \\
\text { salts }\end{array}$ \\
\hline 4 & $\begin{array}{l}\text { baghdad -arab } \\
\text { jbour }\end{array}$ & 19.57 & 2.78 & 27.81 & brown silty clay stiff to medium \\
\hline 5 & $\begin{array}{l}\text { baghdad -arab } \\
\text { jbour }\end{array}$ & 19.57 & 2.8 & 24.23 & brown clay stiff \\
\hline 6 & $\begin{array}{l}\text { baghdad -arab } \\
\text { jbour }\end{array}$ & 18.33 & 2.73 & 29.64 & $\begin{array}{c}\text { brown clayey sandy silt soft to } \\
\text { medium }\end{array}$ \\
\hline 7 & $\begin{array}{l}\text { baghdad -al } \\
\text { wazeriah }\end{array}$ & 18.6 & 2.76 & 30.62 & dark brown silty clay medium \\
\hline 8 & $\begin{array}{l}\text { baghdad -al } \\
\text { wazeriah }\end{array}$ & 20.67 & 2.77 & 23.89 & brown silty clay stiff \\
\hline 9 & $\begin{array}{l}\text { baghdad -al } \\
\text { baladiat }\end{array}$ & 21.01 & 2.8 & 25.49 & brown clay stiff to soft \\
\hline 10 & $\begin{array}{c}\text { baghdad -al } \\
\text { baladiat }\end{array}$ & 18.47 & 2.8 & 25.12 & brown to gray stiff clay \\
\hline 11 & $\begin{array}{l}\text { baghdad -al } \\
\text { baladiat }\end{array}$ & 20.2 & 2.79 & 25.64 & brown silty clay stiff \\
\hline 12 & $\begin{array}{l}\text { baghdad -al } \\
\text { baladiat }\end{array}$ & 20.56 & 2.77 & 28.96 & $\begin{array}{c}\text { brown silty clay to clayey silt } \\
\text { medium to soft }\end{array}$ \\
\hline 13 & $\begin{array}{l}\text { baghdad -al } \\
\text { baladiat }\end{array}$ & 18.63 & 2.76 & 25.76 & brown silty clay with high salts \\
\hline 14 & $\begin{array}{l}\text { baghdad -al } \\
\text { baladiat }\end{array}$ & 19.16 & 2.78 & 34.42 & $\begin{array}{c}\text { black to gray to brown silty clay } \\
\text { to clayey silt medium with high } \\
\text { salt }\end{array}$ \\
\hline 15 & $\begin{array}{l}\text { baghdad -al } \\
\text { baladiat }\end{array}$ & 21.31 & 2.77 & 20.28 & $\begin{array}{l}\text { brown silty clay very stiff with } \\
\text { high salts }\end{array}$ \\
\hline 16 & $\begin{array}{l}\text { baghdad -al } \\
\text { baladiat }\end{array}$ & 16.75 & 2.77 & 25.88 & $\begin{array}{l}\text { black to green to brown silty } \\
\text { clay very stiff with high salt }\end{array}$ \\
\hline 17 & $\begin{array}{l}\text { baghdad -al } \\
\text { baladiat }\end{array}$ & 16.99 & 2.76 & 29.83 & brown clayey silt soft \\
\hline 18 & $\begin{array}{c}\text { baghdad -al } \\
\text { baladiat } \\
\end{array}$ & 20.56 & 2.78 & 24.84 & $\begin{array}{l}\text { black to gray to brown silty clay } \\
\text { stiff with salt } \\
\end{array}$ \\
\hline 19 & $\begin{array}{c}\text { al-sidiah-al } \\
\text { nasireah }\end{array}$ & 20.13 & 2.78 & 23.8 & $\begin{array}{c}\text { brown silty clay to clayey silt } \\
\text { soft }\end{array}$ \\
\hline 20 & $\begin{array}{c}\text { al-sidiah-al } \\
\text { nasireah }\end{array}$ & 20.72 & 2.73 & 24.53 & brown clayey silt \\
\hline 21 & $\begin{array}{l}\text { baghdad -al } \\
\text { baladiat }\end{array}$ & 20.16 & 2.7 & 26.82 & brown silty sand \\
\hline 22 & $\begin{array}{c}\text { al-sidiah-al } \\
\text { nasireah }\end{array}$ & 19.91 & 2.77 & 30.43 & brown silty clay soft \\
\hline 23 & $\begin{array}{c}\text { al-sidiah-al } \\
\text { nasireah }\end{array}$ & 20.72 & 2.77 & 27.13 & $\begin{array}{c}\text { brown silty clay to clayey silt } \\
\text { medium to soft }\end{array}$ \\
\hline 24 & al-mahmodiyah & 19.32 & 2.79 & 24.26 & $\begin{array}{c}\text { brown and gray clay with salt } \\
\text { very stiff }\end{array}$ \\
\hline
\end{tabular}


محلة العلو الهنغسة العد الخلس -2011

\begin{tabular}{|c|c|c|c|c|c|}
\hline 25 & al-mahmodiyah & 22.43 & 2.77 & 23.22 & $\begin{array}{l}\text { silty clay with gravel and root } \\
\text { very stiff with salts }\end{array}$ \\
\hline 26 & al-mahmodiyah & 19.24 & 2.79 & 29.76 & $\begin{array}{c}\text { black to gray clay with salt very } \\
\text { stiff }\end{array}$ \\
\hline 27 & al-mahmodiyah & 18.79 & 2.77 & 25.62 & $\begin{array}{l}\text { gray to brown clay with silt } \\
\text { medium to stiff with root }\end{array}$ \\
\hline 28 & al-mahmodiyah & 16.75 & 2.76 & 23.37 & $\begin{array}{l}\text { gray very stiff clay with silt } \\
\text { with gravel }\end{array}$ \\
\hline 29 & hay tounis & 18.7 & 2.77 & 32.86 & soft brown silty clay \\
\hline 30 & hay tounis & 18.7 & 2.79 & 25.14 & $\begin{array}{l}\text { stiff brown clay with silt with } \\
\text { broken brick }\end{array}$ \\
\hline 31 & $\begin{array}{l}\text { baghdad -al } \\
\text { kmalyah }\end{array}$ & 21.3 & 2.77 & 20.48 & medium brown silty clay \\
\hline 32 & $\begin{array}{l}\text { baghdad -al } \\
\text { kmalyah }\end{array}$ & 20.1 & 2.78 & 23.94 & stiff brown silty clay \\
\hline 33 & $\begin{array}{l}\text { baghdad -al } \\
\text { kmalyah }\end{array}$ & 17.1 & 2.72 & 24.61 & brown clayey silt soft \\
\hline 34 & $\begin{array}{l}\text { baghdad -al } \\
\text { kmalyah }\end{array}$ & 17.5 & 2.74 & 23.73 & $\begin{array}{c}\text { brown clayey silt medium to } \\
\text { soft }\end{array}$ \\
\hline 35 & $\begin{array}{l}\text { baghdad -al } \\
\text { kmalyah }\end{array}$ & 17.8 & 2.75 & 21.65 & brown silty clay stiff to medium \\
\hline 36 & babelonia & 19.16 & 2.78 & 33.39 & soft brown silty clay \\
\hline 37 & babelonia & 20.08 & 2.75 & 23.25 & $\begin{array}{l}\text { medium to stiff brown silty clay } \\
\text { with salts }\end{array}$ \\
\hline 38 & $\begin{array}{c}\text { baghdad-al } \\
\text { kreaght }\end{array}$ & 19.3 & 2.75 & 36.71 & $\begin{array}{c}\text { gray to red to brown silty clay } \\
\text { medium to soft }\end{array}$ \\
\hline 39 & $\begin{array}{l}\text { baghdad-al } \\
\text { kreaght }\end{array}$ & 20.02 & 2.75 & 31.31 & medium red to brown silty clay \\
\hline 40 & $\begin{array}{l}\text { baghdad-al } \\
\text { kreaght }\end{array}$ & 19.22 & 2.78 & 23.39 & $\begin{array}{c}\text { medium to stiff brown silty clay } \\
\text { dry }\end{array}$ \\
\hline 41 & $\begin{array}{l}\text { baghdad-al } \\
\text { kreaght }\end{array}$ & 19 & 2.74 & 36.72 & brown very soft silty clay \\
\hline 42 & naseriah & 21.2 & 2.77 & 23.26 & $\begin{array}{c}\text { brown medium to soft sandy } \\
\text { silty clay with doer }\end{array}$ \\
\hline 43 & naseriah & 22.5 & 2.75 & 25.6 & $\begin{array}{l}\text { medium brown silty clay with } \\
\text { sand }\end{array}$ \\
\hline 44 & $\begin{array}{l}\text { hay al amean- } \\
\text { baghdad }\end{array}$ & 19.73 & 2.8 & 31.21 & $\begin{array}{l}\text { stiff to very stiff brown silty } \\
\text { clay }\end{array}$ \\
\hline 45 & $\begin{array}{l}\text { hay al amean- } \\
\text { baghdad }\end{array}$ & 19.83 & 2.8 & 31.19 & medium to soft brown silty clay \\
\hline 46 & maysan & 17.1 & 2.77 & 66.84 & soft to medium brown silty clay \\
\hline 47 & maysan & 15.92 & 2.77 & 38.32 & soft to medium brown silty clay \\
\hline 48 & wasit & 18.54 & 2.73 & 28.54 & soft to medium clayey silt \\
\hline 49 & maysan & 20.2 & 2.73 & 29.08 & soft gray clayey silt \\
\hline 50 & maysan & 20 & 2.72 & 35.97 & $\begin{array}{l}\text { grey silty clay to clayey silt } \\
\text { medium }\end{array}$ \\
\hline 51 & al latifeah & 21.4 & 2.78 & 27.95 & brown silty clay medium to stiff \\
\hline 52 & al latifeah & 21.28 & 2.79 & 23.9 & $\begin{array}{l}\text { stiff to very stiff grey to brown } \\
\text { silty clay }\end{array}$ \\
\hline 53 & hay al salam & 20.08 & 2.79 & 26.92 & $\begin{array}{l}\text { stiff to very stiff grey to brown } \\
\text { silty clay }\end{array}$ \\
\hline 54 & al-swearah & 19.33 & 2.75 & 18 & brown silty sand with clay \\
\hline
\end{tabular}


Table (2) Summary of Test Results

\begin{tabular}{|c|c|c|c|c|c|c|c|c|c|c|c|c|c|}
\hline \multirow{3}{*}{$\stackrel{\varrho}{\mathrm{E}}$} & \multicolumn{13}{|c|}{ coefficient of permeability $(\mathrm{k})$ in $\mathrm{cm} / \mathrm{sec}$} \\
\hline & \multirow{2}{*}{\multicolumn{2}{|c|}{$\begin{array}{l}\text { by falling } \\
\text { head } \\
\text { method }\end{array}$}} & \multicolumn{7}{|c|}{ by ordinary consolidation method with load in $(\mathrm{kn} / \mathrm{m} 2)$} & \multicolumn{4}{|c|}{$\begin{array}{l}\text { by proposed consolidation method } \\
\text { with load in }(\mathrm{kn} / \mathrm{m} 2)\end{array}$} \\
\hline & & & 10 & 50 & 100 & 200 & & & & 1 & & & 800 \\
\hline 1 & $\begin{array}{c}8.67 \mathrm{E}- \\
08\end{array}$ & $\begin{array}{l}8.89 \mathrm{E}- \\
08\end{array}$ & $\begin{array}{l}4.00 \mathrm{E}- \\
08\end{array}$ & $\begin{array}{l}2.98 \mathrm{E}- \\
08\end{array}$ & $\begin{array}{l}2.50 \mathrm{E}- \\
08\end{array}$ & $\begin{array}{l}1.56 \mathrm{E}- \\
08\end{array}$ & $\begin{array}{c}8.57 \mathrm{E}- \\
09\end{array}$ & $\begin{array}{l}8.63 \mathrm{E}- \\
08\end{array}$ & $\begin{array}{l}15 \mathrm{E}- \\
08\end{array}$ & $\begin{array}{l}3.15 \mathrm{E}- \\
08\end{array}$ & $\begin{array}{l}2.51 \mathrm{E}- \\
08\end{array}$ & $\begin{array}{c}1.52 \mathrm{E}- \\
08\end{array}$ & $\begin{array}{l}8.90 \mathrm{E}- \\
09\end{array}$ \\
\hline 2 & $\begin{array}{c}8.43 \mathrm{E}- \\
08\end{array}$ & $\begin{array}{l}8.53 \mathrm{E}- \\
08\end{array}$ & $\begin{array}{c}5.40 \mathrm{E}- \\
08\end{array}$ & $\begin{array}{c}5.28 \mathrm{E}- \\
08\end{array}$ & $\begin{array}{l}2.95 \mathrm{E}- \\
08\end{array}$ & $\begin{array}{c}1.77 \mathrm{E}- \\
08\end{array}$ & $\begin{array}{c}1.01 \mathrm{E}- \\
08\end{array}$ & $\begin{array}{c}8.04 \mathrm{E}- \\
08\end{array}$ & $\begin{array}{c}5.33 \mathrm{E}- \\
08\end{array}$ & $\begin{array}{c}5.25 \mathrm{E}- \\
08\end{array}$ & $\begin{array}{c}3.03 \mathrm{E}- \\
08\end{array}$ & $\begin{array}{c}1.73 \mathrm{E}- \\
08\end{array}$ & $\begin{array}{c}1.05 \mathrm{E}- \\
08\end{array}$ \\
\hline 3 & $\begin{array}{c}6.90 \mathrm{E}- \\
08\end{array}$ & $\begin{array}{c}6.51 \mathrm{E}- \\
08\end{array}$ & $\begin{array}{c}5.67 \mathrm{E}- \\
08\end{array}$ & $\begin{array}{c}4.37 \mathrm{E}- \\
08\end{array}$ & $\begin{array}{l}2.57 \mathrm{E}- \\
08\end{array}$ & $\begin{array}{c}9.42 \mathrm{E}- \\
09\end{array}$ & $\begin{array}{l}6.25 \mathrm{E}- \\
09\end{array}$ & $\begin{array}{c}6.33 \mathrm{E}- \\
08\end{array}$ & $\begin{array}{l}5.62 \mathrm{E}- \\
08\end{array}$ & $\begin{array}{c}4.42 \mathrm{E}- \\
08\end{array}$ & $\begin{array}{l}2.64 \mathrm{E}- \\
08\end{array}$ & $\begin{array}{c}9.67 \mathrm{E}- \\
09\end{array}$ & $\begin{array}{c}6.17 \mathrm{E}- \\
09\end{array}$ \\
\hline 4 & $\begin{array}{c}4.40 \mathrm{E}- \\
07\end{array}$ & $\begin{array}{l}4.32 \mathrm{E}- \\
07\end{array}$ & $\begin{array}{c}3.91 \mathrm{E}- \\
07\end{array}$ & $\begin{array}{l}4.16 \mathrm{E}- \\
08\end{array}$ & $\begin{array}{l}2.17 \mathrm{E}- \\
08\end{array}$ & $\begin{array}{l}1.38 \mathrm{E}- \\
08\end{array}$ & $\begin{array}{c}7.93 \mathrm{E}- \\
09\end{array}$ & $\begin{array}{c}4.19 \mathrm{E}- \\
07\end{array}$ & $\begin{array}{c}3.40 \mathrm{E}- \\
07\end{array}$ & $\begin{array}{c}4.10 \mathrm{E}- \\
08\end{array}$ & $\begin{array}{c}2.27 \mathrm{E}- \\
08\end{array}$ & $\begin{array}{c}1.20 \mathrm{E}- \\
08\end{array}$ & $\begin{array}{c}7.91 \mathrm{E}- \\
09\end{array}$ \\
\hline 5 & $\begin{array}{c}5.80 \mathrm{E}- \\
08\end{array}$ & $\begin{array}{c}6.98 \mathrm{E}- \\
08\end{array}$ & $\begin{array}{c}6.28 \mathrm{E}- \\
08\end{array}$ & $\begin{array}{c}1.99 \mathrm{E}- \\
08\end{array}$ & $\begin{array}{c}1.16 \mathrm{E}- \\
08\end{array}$ & $\begin{array}{c}1.03 \mathrm{E}- \\
08\end{array}$ & $\begin{array}{c}9.82 \mathrm{E}- \\
09\end{array}$ & $\begin{array}{c}7.12 \mathrm{E}- \\
08\end{array}$ & $\begin{array}{c}\text { 6.79E- } \\
08\end{array}$ & $\begin{array}{c}1.97 \mathrm{E}- \\
08\end{array}$ & $\begin{array}{c}1.18 \mathrm{E}- \\
08\end{array}$ & $\begin{array}{c}1.02 \mathrm{E}- \\
08\end{array}$ & $\begin{array}{c}9.99 \mathrm{E}- \\
09\end{array}$ \\
\hline 6 & $\begin{array}{c}1.60 \mathrm{E}- \\
07\end{array}$ & $\begin{array}{c}1.86 \mathrm{E}- \\
07\end{array}$ & $\begin{array}{c}9.32 \mathrm{E}- \\
08\end{array}$ & $\begin{array}{c}4.57 \mathrm{E}- \\
08\end{array}$ & $\begin{array}{c}3.19 \mathrm{E}- \\
08\end{array}$ & $\begin{array}{c}2.18 \mathrm{E}- \\
08\end{array}$ & $\begin{array}{c}1.39 \mathrm{E}- \\
08\end{array}$ & $\begin{array}{c}1.75 \mathrm{E}- \\
07\end{array}$ & $\begin{array}{c}9.81 \mathrm{E}- \\
08\end{array}$ & $\begin{array}{c}4.68 \mathrm{E}- \\
08\end{array}$ & $\begin{array}{c}3.13 \mathrm{E}- \\
08\end{array}$ & $\begin{array}{c}2.10 \mathrm{E}- \\
08\end{array}$ & $\begin{array}{c}1.36 \mathrm{E}- \\
08\end{array}$ \\
\hline 7 & $\begin{array}{c}5.50 \mathrm{E}- \\
08\end{array}$ & $\begin{array}{c}5.73 \mathrm{E}- \\
08\end{array}$ & $\begin{array}{c}4.65 \mathrm{E}- \\
08\end{array}$ & $\begin{array}{c}3.63 \mathrm{E}- \\
08\end{array}$ & $\begin{array}{c}2.23 \mathrm{E}- \\
08\end{array}$ & $\begin{array}{c}1.28 \mathrm{E}- \\
08\end{array}$ & $\begin{array}{c}\text { 7.63E- } \\
09\end{array}$ & $\begin{array}{c}5.91 \mathrm{E}- \\
08\end{array}$ & $\begin{array}{c}4.64 \mathrm{E}- \\
08\end{array}$ & $\begin{array}{c}\text { 3.74E- } \\
08\end{array}$ & $\begin{array}{c}2.24 \mathrm{E}- \\
08\end{array}$ & $\begin{array}{c}1.22 \mathrm{E}- \\
08\end{array}$ & $\begin{array}{c}7.67 \mathrm{E}- \\
09\end{array}$ \\
\hline 8 & $\begin{array}{c}7.21 \mathrm{E}- \\
08\end{array}$ & $\begin{array}{c}7.38 \mathrm{E}- \\
08\end{array}$ & $\begin{array}{c}4.43 \mathrm{E}- \\
08 \\
\end{array}$ & $\begin{array}{c}2.31 \mathrm{E}- \\
08\end{array}$ & $\begin{array}{c}2.21 \mathrm{E}- \\
08\end{array}$ & $\begin{array}{c}1.41 \mathrm{E}- \\
08\end{array}$ & $\begin{array}{c}9.04 \mathrm{E}- \\
09\end{array}$ & $\begin{array}{c}7.14 \mathrm{E}- \\
08\end{array}$ & $\begin{array}{c}4.23 \mathrm{E}- \\
08\end{array}$ & $\begin{array}{c}2.30 \mathrm{E}- \\
08\end{array}$ & $\begin{array}{c}2.25 \mathrm{E}- \\
08\end{array}$ & $\begin{array}{c}1.48 \mathrm{E}- \\
08\end{array}$ & $\begin{array}{c}9.09 \mathrm{E}- \\
09\end{array}$ \\
\hline 9 & $\begin{array}{c}6.80 \mathrm{E}- \\
08\end{array}$ & $\begin{array}{c}7.30 \mathrm{E}- \\
08\end{array}$ & \begin{tabular}{|c}
$5.35 \mathrm{E}-$ \\
08 \\
\end{tabular} & $\begin{array}{c}3.69 \mathrm{E}- \\
08\end{array}$ & $\begin{array}{c}2.35 \mathrm{E}- \\
08\end{array}$ & $\begin{array}{c}1.57 \mathrm{E}- \\
08\end{array}$ & $\begin{array}{c}9.26 \mathrm{E}- \\
09\end{array}$ & $\begin{array}{c}7.10 \mathrm{E}- \\
08\end{array}$ & $\begin{array}{c}5.53 \mathrm{E}- \\
08\end{array}$ & $\begin{array}{c}3.62 \mathrm{E}- \\
08\end{array}$ & $\begin{array}{c}2.37 \mathrm{E}- \\
08\end{array}$ & $\begin{array}{c}1.54 \mathrm{E}- \\
08\end{array}$ & $\begin{array}{c}9.37 \mathrm{E}- \\
09\end{array}$ \\
\hline 10 & $\begin{array}{c}6.57 \mathrm{E}- \\
08\end{array}$ & $\begin{array}{c}7.00 \mathrm{E}- \\
08\end{array}$ & $\begin{array}{c}5.24 \mathrm{E}- \\
08\end{array}$ & $\begin{array}{c}4.58 \mathrm{E}- \\
08\end{array}$ & $\begin{array}{c}3.36 \mathrm{E}- \\
08\end{array}$ & $\begin{array}{c}1.51 \mathrm{E}- \\
08\end{array}$ & $\begin{array}{c}\text { 7.73E- } \\
09\end{array}$ & $\begin{array}{c}7.07 \mathrm{E}- \\
08\end{array}$ & $\begin{array}{c}5.23 \mathrm{E}- \\
08\end{array}$ & $\begin{array}{c}4.58 \mathrm{E}- \\
08\end{array}$ & $\begin{array}{c}3.38 \mathrm{E}- \\
08\end{array}$ & $\begin{array}{c}1.50 \mathrm{E}- \\
08\end{array}$ & $\begin{array}{c}7.87 \mathrm{E}- \\
09\end{array}$ \\
\hline 11 & $\begin{array}{c}5.30 \mathrm{E}- \\
08\end{array}$ & $\begin{array}{c}4.58 \mathrm{E}- \\
08\end{array}$ & $\begin{array}{c}3.62 \mathrm{E}- \\
08\end{array}$ & $\begin{array}{c}1.90 \mathrm{E}- \\
08\end{array}$ & $\begin{array}{c}1.47 \mathrm{E}- \\
08\end{array}$ & $\begin{array}{c}9.92 \mathrm{E}- \\
09\end{array}$ & $\begin{array}{c}\text { 6.92E- } \\
09\end{array}$ & $\begin{array}{c}4.53 \mathrm{E}- \\
08\end{array}$ & $\begin{array}{c}3.67 \mathrm{E}- \\
08\end{array}$ & $\begin{array}{c}1.94 \mathrm{E}- \\
08\end{array}$ & $\begin{array}{c}1.43 \mathrm{E}- \\
08\end{array}$ & $\begin{array}{c}9.97 \mathrm{E}- \\
09\end{array}$ & $\begin{array}{c}6.91 \mathrm{E}- \\
09\end{array}$ \\
\hline 12 & $6.12 \mathrm{E}-$ & $\begin{array}{c}5.65 \mathrm{E}- \\
08\end{array}$ & $\begin{array}{c}4.29 \mathrm{E}- \\
08\end{array}$ & $\begin{array}{c}2.69 \mathrm{E}- \\
08\end{array}$ & $\begin{array}{c}2.55 \mathrm{E}- \\
08\end{array}$ & $\begin{array}{c}1.76 \mathrm{E}- \\
08\end{array}$ & $\begin{array}{c}1.46 \mathrm{E}- \\
08\end{array}$ & $\begin{array}{c}5.56 \mathrm{E}- \\
08\end{array}$ & $\begin{array}{c}4.21 \mathrm{E}- \\
08\end{array}$ & $\begin{array}{c}2.66 \mathrm{E}- \\
08\end{array}$ & $\begin{array}{c}2.54 \mathrm{E}- \\
08\end{array}$ & $\begin{array}{c}1.83 \mathrm{E}- \\
08\end{array}$ & $\begin{array}{c}1.47 \mathrm{E}- \\
08\end{array}$ \\
\hline 13 & $\begin{array}{c}7.27 \mathrm{E}- \\
08\end{array}$ & $\begin{array}{c}7.55 \mathrm{E}- \\
08\end{array}$ & $\begin{array}{c}6.55 \mathrm{E}- \\
08\end{array}$ & $\begin{array}{c}2.11 \mathrm{E}- \\
08\end{array}$ & $\begin{array}{c}1.17 \mathrm{E}- \\
08\end{array}$ & $\begin{array}{c}6.89 \mathrm{E}- \\
09\end{array}$ & $\begin{array}{c}3.82 \mathrm{E}- \\
09\end{array}$ & $\begin{array}{c}\text { 7.64E- } \\
08\end{array}$ & $\begin{array}{c}6.64 \mathrm{E}- \\
08\end{array}$ & $\begin{array}{c}2.18 \mathrm{E}- \\
08\end{array}$ & $\begin{array}{c}1.17 \mathrm{E}- \\
08\end{array}$ & $\begin{array}{c}6.74 \mathrm{E}- \\
09\end{array}$ & $\begin{array}{c}3.81 \mathrm{E}- \\
09\end{array}$ \\
\hline 14 & $\begin{array}{c}8.07 \mathrm{E}- \\
07\end{array}$ & $\begin{array}{c}6.63 \mathrm{E}- \\
07\end{array}$ & $\begin{array}{c}3.26 \mathrm{E}- \\
08\end{array}$ & $\begin{array}{c}2.75 \mathrm{E}- \\
08\end{array}$ & $\begin{array}{c}2.00 \mathrm{E}- \\
08\end{array}$ & $\begin{array}{c}1.68 \mathrm{E}- \\
08\end{array}$ & $\begin{array}{c}1.16 \mathrm{E}- \\
08\end{array}$ & $\begin{array}{c}6.81 \mathrm{E}- \\
07\end{array}$ & $\begin{array}{c}3.30 \mathrm{E}- \\
08\end{array}$ & $\begin{array}{c}2.60 \mathrm{E}- \\
08\end{array}$ & $\begin{array}{l}04 \mathrm{E}- \\
08\end{array}$ & $\begin{array}{c}1.71 \mathrm{E}- \\
08\end{array}$ & $\begin{array}{l}1.23 \mathrm{E}- \\
08\end{array}$ \\
\hline 15 & $\begin{array}{c}6.00 \mathrm{E}- \\
08\end{array}$ & $\begin{array}{c}4.61 \mathrm{E}- \\
08\end{array}$ & $\begin{array}{c}4.36 \mathrm{E}- \\
08\end{array}$ & $\begin{array}{c}4.23 \mathrm{E}- \\
08\end{array}$ & $\begin{array}{c}2.01 \mathrm{E}- \\
08\end{array}$ & $\begin{array}{c}6.83 \mathrm{E}- \\
09\end{array}$ & $\begin{array}{c}3.65 \mathrm{E}- \\
09\end{array}$ & $\begin{array}{c}4.57 \mathrm{E}- \\
08\end{array}$ & $\begin{array}{c}4.45 \mathrm{E}- \\
08\end{array}$ & $\begin{array}{c}4.44 \mathrm{E}- \\
08\end{array}$ & $\begin{array}{c}1.95 \mathrm{E}- \\
08\end{array}$ & $\begin{array}{c}6.64 \mathrm{E}- \\
09\end{array}$ & $\begin{array}{c}3.62 \mathrm{E}- \\
09\end{array}$ \\
\hline 16 & $\begin{array}{c}5.50 \mathrm{E}- \\
08\end{array}$ & $\begin{array}{c}6.89 \mathrm{E}- \\
08\end{array}$ & \begin{tabular}{|c|}
$5.18 \mathrm{E}-$ \\
08 \\
\end{tabular} & $\begin{array}{c}4.69 \mathrm{E}- \\
08\end{array}$ & $\begin{array}{c}3.28 \mathrm{E}- \\
08\end{array}$ & $\begin{array}{c}1.45 \mathrm{E}- \\
08\end{array}$ & $\begin{array}{c}6.08 \mathrm{E}- \\
09\end{array}$ & $\begin{array}{c}6.86 \mathrm{E}- \\
08\end{array}$ & $\begin{array}{c}5.19 \mathrm{E}- \\
08\end{array}$ & $\begin{array}{c}4.67 \mathrm{E}- \\
08\end{array}$ & $\begin{array}{c}3.14 \mathrm{E}- \\
08\end{array}$ & $\begin{array}{c}1.42 \mathrm{E}- \\
08\end{array}$ & $\begin{array}{c}6.14 \mathrm{E}- \\
09\end{array}$ \\
\hline 17 & $6.35 \mathrm{E}-$ & $\begin{array}{c}6.72 \mathrm{E}- \\
07\end{array}$ & $\begin{array}{c}2.68 \mathrm{E}- \\
08\end{array}$ & $\begin{array}{c}2.60 \mathrm{E}- \\
08\end{array}$ & $\begin{array}{c}2.04 \mathrm{E}- \\
08\end{array}$ & $\begin{array}{c}1.47 \mathrm{E}- \\
08\end{array}$ & $\begin{array}{c}8.57 \mathrm{E}- \\
09\end{array}$ & $\begin{array}{c}6.51 \mathrm{E}- \\
07\end{array}$ & $\begin{array}{c}2.74 \mathrm{E}- \\
08\end{array}$ & $\begin{array}{c}2.66 \mathrm{E}- \\
08\end{array}$ & $\begin{array}{c}2.00 \mathrm{E}- \\
08\end{array}$ & $\begin{array}{c}1.48 \mathrm{E}- \\
08\end{array}$ & $\begin{array}{c}8.97 \mathrm{E}- \\
09\end{array}$ \\
\hline 18 & $2.71 \mathrm{E}-$ & $\begin{array}{c}2.82 \mathrm{E}- \\
08\end{array}$ & E- & $\begin{array}{l}12 \mathrm{E}- \\
08\end{array}$ & $\begin{array}{l}36 \mathrm{E}- \\
08\end{array}$ & $\begin{array}{c}9.30 \mathrm{E}- \\
09\end{array}$ & $\begin{array}{c}7.54 \mathrm{E}- \\
09\end{array}$ & $\begin{array}{c}2.78 \mathrm{E}- \\
08\end{array}$ & $\begin{array}{l}28 \mathrm{E}- \\
08\end{array}$ & $\begin{array}{c}2.01 \mathrm{E}- \\
08\end{array}$ & $\begin{array}{l}35 \mathrm{E}- \\
08\end{array}$ & $\begin{array}{c}.01 \mathrm{E}- \\
09\end{array}$ & $\begin{array}{c}7.49 \mathrm{E}- \\
09\end{array}$ \\
\hline 19 & $\begin{array}{c}1.32 \mathrm{E}- \\
07\end{array}$ & $\begin{array}{c}1.59 \mathrm{E}- \\
07\end{array}$ & $\begin{array}{c}3.02 \mathrm{E}- \\
08 \\
\end{array}$ & $\begin{array}{c}1.76 \mathrm{E}- \\
08\end{array}$ & $\begin{array}{c}1.24 \mathrm{E}- \\
08\end{array}$ & $\begin{array}{c}1.21 \mathrm{E}- \\
08\end{array}$ & $\begin{array}{c}1.17 \mathrm{E}- \\
08\end{array}$ & $\begin{array}{c}1.37 \mathrm{E}- \\
07\end{array}$ & $\begin{array}{c}3.14 \mathrm{E}- \\
08\end{array}$ & $\begin{array}{c}1.74 \mathrm{E}- \\
08\end{array}$ & $\begin{array}{c}1.31 \mathrm{E}- \\
08\end{array}$ & $\begin{array}{c}1.23 \mathrm{E}- \\
08\end{array}$ & $\begin{array}{c}1.13 \mathrm{E}- \\
08\end{array}$ \\
\hline 20 & $\begin{array}{c}8.85 \mathrm{E}- \\
07\end{array}$ & $\begin{array}{c}7.60 \mathrm{E}- \\
07\end{array}$ & $\begin{array}{c}6.81 \mathrm{E}- \\
08\end{array}$ & $\begin{array}{c}3.32 \mathrm{E}- \\
08\end{array}$ & $\begin{array}{c}3.10 \mathrm{E}- \\
08\end{array}$ & $\begin{array}{c}1.84 \mathrm{E}- \\
08\end{array}$ & $\begin{array}{c}1.19 \mathrm{E}- \\
08\end{array}$ & $\begin{array}{c}7.78 \mathrm{E}- \\
07\end{array}$ & $\begin{array}{c}6.47 \mathrm{E}- \\
08\end{array}$ & $\begin{array}{c}3.35 \mathrm{E}- \\
08\end{array}$ & $\begin{array}{c}3.20 \mathrm{E}- \\
08\end{array}$ & $\begin{array}{c}1.80 \mathrm{E}- \\
08\end{array}$ & $\begin{array}{c}1.15 \mathrm{E}- \\
08\end{array}$ \\
\hline & $\begin{array}{c}3.35 \mathrm{E}- \\
06\end{array}$ & $\begin{array}{c}2.51 \mathrm{E}- \\
06\end{array}$ & $\begin{array}{c}3.46 \mathrm{E}- \\
08\end{array}$ & $\begin{array}{c}1.69 \mathrm{E}- \\
08\end{array}$ & $\begin{array}{c}1.08 \mathrm{E}- \\
08\end{array}$ & $\begin{array}{c}6.66 \mathrm{E}- \\
09\end{array}$ & $\begin{array}{c}4.38 \mathrm{E}- \\
09\end{array}$ & $\begin{array}{c}2.51 \mathrm{E}- \\
06\end{array}$ & $\begin{array}{c}\text { 3.32E- } \\
08\end{array}$ & $\begin{array}{c}1.70 \mathrm{E}- \\
08\end{array}$ & $\begin{array}{c}1.09 \mathrm{E}- \\
08\end{array}$ & $\begin{array}{c}6.31 \mathrm{E}- \\
09\end{array}$ & $\begin{array}{c}4.05 \mathrm{E}- \\
09\end{array}$ \\
\hline & $\begin{array}{c}4.80 \mathrm{E}- \\
08\end{array}$ & $\begin{array}{c}3.00 \mathrm{E}- \\
08\end{array}$ & $\begin{array}{c}2.96 \mathrm{E}- \\
08\end{array}$ & $\begin{array}{c}2.00 \mathrm{E}- \\
08\end{array}$ & $\begin{array}{c}1.87 \mathrm{E}- \\
08\end{array}$ & $\begin{array}{c}1.48 \mathrm{E}- \\
08\end{array}$ & $\begin{array}{c}9.71 \mathrm{E}- \\
09\end{array}$ & $\begin{array}{c}3.04 \mathrm{E}- \\
08\end{array}$ & $\begin{array}{c}2.90 \mathrm{E}- \\
08\end{array}$ & $\begin{array}{c}1.95 \mathrm{E}- \\
08\end{array}$ & $\begin{array}{c}1.85 \mathrm{E}- \\
08\end{array}$ & $\begin{array}{c}1.49 \mathrm{E}- \\
08\end{array}$ & $\begin{array}{c}9.65 \mathrm{E}- \\
09\end{array}$ \\
\hline 3 & $\begin{array}{c}4.46 \mathrm{E}- \\
08\end{array}$ & $\begin{array}{c}.38 \mathrm{E}- \\
08 \\
\end{array}$ & $\begin{array}{c}2.86 \mathrm{E}- \\
08 \\
\end{array}$ & $\begin{array}{c}1.91 \mathrm{E}- \\
08\end{array}$ & $\begin{array}{c}1.25 \mathrm{E}- \\
08\end{array}$ & $\begin{array}{c}9.33 \mathrm{E}- \\
09\end{array}$ & $\begin{array}{c}6.09 \mathrm{E}- \\
09\end{array}$ & $\begin{array}{c}4.32 \mathrm{E}- \\
08\end{array}$ & $\begin{array}{c}2.82 \mathrm{E}- \\
08\end{array}$ & $\begin{array}{c}1.93 \mathrm{E}- \\
08\end{array}$ & $\begin{array}{c}1.28 \mathrm{E}- \\
08\end{array}$ & $\begin{array}{c}9.39 \mathrm{E}- \\
09\end{array}$ & $\begin{array}{c}5.80 \mathrm{E}- \\
09\end{array}$ \\
\hline & $3.06 \mathrm{E}-$ & $2.70 \mathrm{E}-$ & $3.71 \mathrm{E}-$ & $3.40 \mathrm{E}-$ & $3.29 \mathrm{E}-$ & $\begin{array}{c}\text { 3.19E- } \\
09\end{array}$ & $\begin{array}{c}2.94 \mathrm{E}- \\
09\end{array}$ & $\begin{array}{c}2.74 \mathrm{E}- \\
08\end{array}$ & $\begin{array}{c}3.76 \mathrm{E}- \\
08\end{array}$ & $\begin{array}{c}3.30 \mathrm{E}- \\
09\end{array}$ & $\begin{array}{c}3.21 \mathrm{E}- \\
09\end{array}$ & $\begin{array}{c}3.18 \mathrm{E}- \\
09\end{array}$ & $\begin{array}{c}3.05 \mathrm{E}- \\
09\end{array}$ \\
\hline & $\begin{array}{c}3.30 \mathrm{E}- \\
08\end{array}$ & $\begin{array}{c}2.35 \mathrm{E}- \\
08\end{array}$ & $\begin{array}{c}2.06 \mathrm{E}- \\
08\end{array}$ & $\begin{array}{c}1.80 \mathrm{E}- \\
08\end{array}$ & $\begin{array}{c}1.30 \mathrm{E}- \\
08\end{array}$ & $\begin{array}{c}1.13 \mathrm{E}- \\
08\end{array}$ & $\begin{array}{c}6.42 \mathrm{E}- \\
09\end{array}$ & $\begin{array}{c}2.33 \mathrm{E}- \\
08\end{array}$ & $\begin{array}{c}2.06 \mathrm{E}- \\
08\end{array}$ & $\begin{array}{c}1.85 \mathrm{E}- \\
08\end{array}$ & $\begin{array}{c}1.27 \mathrm{E}- \\
08\end{array}$ & $\begin{array}{c}1.14 \mathrm{E}- \\
08\end{array}$ & $\begin{array}{l}55 \mathrm{E}- \\
09\end{array}$ \\
\hline 6 & $\begin{array}{c}2.90 \mathrm{E}- \\
08\end{array}$ & $\begin{array}{c}2.85 \mathrm{E}- \\
08\end{array}$ & $\begin{array}{c}2.28 \mathrm{E}- \\
08\end{array}$ & $\begin{array}{c}1.88 \mathrm{E}- \\
08\end{array}$ & $\begin{array}{c}1.51 \mathrm{E}- \\
08\end{array}$ & $\begin{array}{c}1.16 \mathrm{E}- \\
08\end{array}$ & $\begin{array}{c}5.02 \mathrm{E}- \\
09\end{array}$ & $\begin{array}{c}2.63 \mathrm{E}- \\
08\end{array}$ & $\begin{array}{c}2.10 \mathrm{E}- \\
08\end{array}$ & $\begin{array}{c}1.82 \mathrm{E}- \\
08\end{array}$ & $\begin{array}{c}1.53 \mathrm{E}- \\
08\end{array}$ & $\begin{array}{c}1.17 \mathrm{E}- \\
08\end{array}$ & $\begin{array}{c}5.14 \mathrm{E}- \\
09\end{array}$ \\
\hline & $\begin{array}{c}5.00 \mathrm{E}- \\
08\end{array}$ & $\begin{array}{c}4.56 \mathrm{E}- \\
08\end{array}$ & $\begin{array}{c}3.98 \mathrm{E}- \\
08\end{array}$ & $\begin{array}{c}2.12 \mathrm{E}- \\
08\end{array}$ & $\begin{array}{c}1.96 \mathrm{E}- \\
08\end{array}$ & $\begin{array}{c}1.39 \mathrm{E}- \\
08\end{array}$ & $\begin{array}{c}5.53 \mathrm{E}- \\
09\end{array}$ & $\begin{array}{c}4.32 \mathrm{E}- \\
08\end{array}$ & $\begin{array}{c}3.76 \mathrm{E}- \\
08\end{array}$ & $\begin{array}{c}2.11 \mathrm{E}- \\
08\end{array}$ & $\begin{array}{c}2.10 \mathrm{E}- \\
08\end{array}$ & $\begin{array}{c}1.30 \mathrm{E}- \\
08\end{array}$ & $\begin{array}{c}6.40 \mathrm{E}- \\
09\end{array}$ \\
\hline
\end{tabular}


Table (2) Summary of Test Results (continue)

\begin{tabular}{|c|c|c|c|c|c|c|c|c|c|c|c|c|c|}
\hline \multirow{3}{*}{ 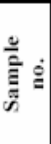 } & \multicolumn{13}{|c|}{ coefficient of permeability $(\mathrm{k})$ in $\mathrm{cm} / \mathrm{sec}$} \\
\hline & \multirow{2}{*}{$\begin{array}{c}\text { by } \\
\text { falling } \\
\text { head } \\
\text { method }\end{array}$} & \multicolumn{6}{|c|}{ by ordinary consolidation method with load in ( $\mathrm{kn} / \mathrm{m} 2)$} & \multicolumn{6}{|c|}{ by proposed consolidation method with load in ( $\mathrm{kn} / \mathrm{m} 2)$} \\
\hline & & 10 & 50 & 100 & 200 & 400 & 800 & 10 & 50 & 100 & 200 & 400 & 800 \\
\hline 28 & $\begin{array}{c}4.20 \mathrm{E}- \\
08\end{array}$ & $\begin{array}{c}4.88 \mathrm{E}- \\
08\end{array}$ & $\begin{array}{c}8.00 \mathrm{E}- \\
09\end{array}$ & $\begin{array}{c}7.37 \mathrm{E}- \\
09\end{array}$ & $\begin{array}{c}5.88 \mathrm{E}- \\
09\end{array}$ & $\begin{array}{c}4.79 \mathrm{E}- \\
09\end{array}$ & $\begin{array}{c}4.23 \mathrm{E}- \\
09\end{array}$ & $\begin{array}{c}5.06 \mathrm{E}- \\
08\end{array}$ & $\begin{array}{c}8.17 \mathrm{E}- \\
09\end{array}$ & $\begin{array}{c}7.02 \mathrm{E}- \\
09\end{array}$ & $\begin{array}{c}5.75 \mathrm{E}- \\
09\end{array}$ & $\begin{array}{l}4.71 \mathrm{E}- \\
09\end{array}$ & $\begin{array}{c}4.22 \mathrm{E}- \\
09\end{array}$ \\
\hline 29 & $\begin{array}{c}3.10 \mathrm{E}- \\
08\end{array}$ & $\begin{array}{c}5.69 \mathrm{E}- \\
08\end{array}$ & $\begin{array}{c}4.42 \mathrm{E}- \\
08\end{array}$ & $\begin{array}{c}2.27 \mathrm{E}- \\
08\end{array}$ & $\begin{array}{c}1.49 \mathrm{E}- \\
08\end{array}$ & $\begin{array}{c}9.32 \mathrm{E}- \\
09\end{array}$ & $\begin{array}{c}4.65 \mathrm{E}- \\
09\end{array}$ & $\begin{array}{c}5.86 \mathrm{E}- \\
08\end{array}$ & $\begin{array}{c}4.45 \mathrm{E}- \\
08\end{array}$ & $\begin{array}{c}2.26 \mathrm{E}- \\
08\end{array}$ & $\begin{array}{c}1.46 \mathrm{E}- \\
08\end{array}$ & $\begin{array}{c}9.22 \mathrm{E}- \\
09\end{array}$ & $\begin{array}{c}4.67 \mathrm{E}- \\
09\end{array}$ \\
\hline 30 & $\begin{array}{c}2.80 \mathrm{E}- \\
08\end{array}$ & $\begin{array}{c}3.55 \mathrm{E}- \\
08\end{array}$ & $\begin{array}{c}1.80 \mathrm{E}- \\
08\end{array}$ & $\begin{array}{c}1.24 \mathrm{E}- \\
08\end{array}$ & $\begin{array}{c}6.76 \mathrm{E}- \\
09\end{array}$ & $\begin{array}{c}5.60 \mathrm{E}- \\
09\end{array}$ & $\begin{array}{c}4.08 \mathrm{E}- \\
09\end{array}$ & $\begin{array}{c}3.51 \mathrm{E}- \\
08\end{array}$ & $\begin{array}{c}1.89 \mathrm{E}- \\
08\end{array}$ & $\begin{array}{c}1.20 \mathrm{E}- \\
08\end{array}$ & $\begin{array}{c}7.93 \mathrm{E}- \\
09\end{array}$ & $\begin{array}{c}5.74 \mathrm{E}- \\
09\end{array}$ & $\begin{array}{c}3.83 \mathrm{E}- \\
09\end{array}$ \\
\hline 31 & $\begin{array}{c}4.12 \mathrm{E}- \\
08\end{array}$ & $\begin{array}{c}3.91 \mathrm{E}- \\
08\end{array}$ & $\begin{array}{c}3.21 \mathrm{E}- \\
08\end{array}$ & $\begin{array}{c}2.60 \mathrm{E}- \\
08\end{array}$ & $\begin{array}{c}2.21 \mathrm{E}- \\
08\end{array}$ & $\begin{array}{c}1.56 \mathrm{E}- \\
08\end{array}$ & $\begin{array}{c}1.06 \mathrm{E}- \\
08\end{array}$ & $\begin{array}{c}3.98 \mathrm{E}- \\
08\end{array}$ & $\begin{array}{c}3.17 \mathrm{E}- \\
08\end{array}$ & $\begin{array}{c}2.53 \mathrm{E}- \\
08\end{array}$ & $\begin{array}{c}2.22 \mathrm{E}- \\
08\end{array}$ & $\begin{array}{c}1.53 \mathrm{E}- \\
08\end{array}$ & $\begin{array}{c}1.02 \mathrm{E}- \\
08\end{array}$ \\
\hline 32 & $\begin{array}{c}5.16 \mathrm{E}- \\
08\end{array}$ & $\begin{array}{c}5.04 \mathrm{E}- \\
08\end{array}$ & $\begin{array}{c}3.14 \mathrm{E}- \\
08\end{array}$ & $\begin{array}{c}2.23 \mathrm{E}- \\
08\end{array}$ & $\begin{array}{c}2.11 \mathrm{E}- \\
08\end{array}$ & $\begin{array}{c}1.04 \mathrm{E}- \\
08\end{array}$ & $\begin{array}{c}4.93 \mathrm{E}- \\
09\end{array}$ & $\begin{array}{c}5.10 \mathrm{E}- \\
08\end{array}$ & $\begin{array}{c}3.26 \mathrm{E}- \\
08\end{array}$ & $\begin{array}{c}2.23 \mathrm{E}- \\
08\end{array}$ & $\begin{array}{c}2.11 \mathrm{E}- \\
08\end{array}$ & $\begin{array}{c}1.04 \mathrm{E}- \\
08\end{array}$ & $\begin{array}{c}5.07 \mathrm{E}- \\
09\end{array}$ \\
\hline 33 & $\begin{array}{c}7.90 \mathrm{E}- \\
08\end{array}$ & $\begin{array}{c}7.46 \mathrm{E}- \\
08\end{array}$ & $\begin{array}{c}5.75 \mathrm{E}- \\
08\end{array}$ & $\begin{array}{c}3.86 \mathrm{E}- \\
08\end{array}$ & $\begin{array}{c}2.76 \mathrm{E}- \\
08\end{array}$ & $\begin{array}{c}1.68 \mathrm{E}- \\
08\end{array}$ & $\begin{array}{c}1.04 \mathrm{E}- \\
08\end{array}$ & $\begin{array}{c}7.54 \mathrm{E}- \\
08\end{array}$ & $\begin{array}{c}6.12 \mathrm{E}- \\
08\end{array}$ & $\begin{array}{c}3.92 \mathrm{E}- \\
08\end{array}$ & $\begin{array}{c}2.71 \mathrm{E}- \\
08\end{array}$ & $\begin{array}{c}1.76 \mathrm{E}- \\
08\end{array}$ & $\begin{array}{c}1.05 \mathrm{E}- \\
08\end{array}$ \\
\hline 34 & $\begin{array}{c}8.80 \mathrm{E}- \\
08\end{array}$ & $\begin{array}{c}8.91 \mathrm{E}- \\
08\end{array}$ & $\begin{array}{c}2.07 \mathrm{E}- \\
08\end{array}$ & $\begin{array}{c}1.60 \mathrm{E}- \\
08\end{array}$ & $\begin{array}{c}1.48 \mathrm{E}- \\
08\end{array}$ & $\begin{array}{c}5.72 \mathrm{E}- \\
09\end{array}$ & $\begin{array}{c}5.25 \mathrm{E}- \\
09\end{array}$ & $\begin{array}{c}8.53 \mathrm{E}- \\
08\end{array}$ & $\begin{array}{c}2.04 \mathrm{E}- \\
08\end{array}$ & $\begin{array}{c}1.52 \mathrm{E}- \\
08\end{array}$ & $\begin{array}{c}1.47 \mathrm{E}- \\
08\end{array}$ & $\begin{array}{c}5.54 \mathrm{E}- \\
09\end{array}$ & $\begin{array}{c}5.37 \mathrm{E}- \\
09\end{array}$ \\
\hline 35 & $\begin{array}{c}6.34 \mathrm{E}- \\
08\end{array}$ & $\begin{array}{c}5.77 \mathrm{E}- \\
08\end{array}$ & $\begin{array}{c}4.42 \mathrm{E}- \\
08\end{array}$ & $\begin{array}{c}1.69 \mathrm{E}- \\
08\end{array}$ & $\begin{array}{c}1.64 \mathrm{E}- \\
08\end{array}$ & $\begin{array}{c}8.87 \mathrm{E}- \\
09\end{array}$ & $\begin{array}{c}5.59 \mathrm{E}- \\
09\end{array}$ & $\begin{array}{c}5.07 \mathrm{E}- \\
08\end{array}$ & $\begin{array}{c}3.89 \mathrm{E}- \\
08\end{array}$ & $\begin{array}{c}1.70 \mathrm{E}- \\
08\end{array}$ & $\begin{array}{c}1.63 \mathrm{E}- \\
08\end{array}$ & $\begin{array}{c}8.99 \mathrm{E}- \\
09\end{array}$ & $\begin{array}{c}5.53 \mathrm{E}- \\
09\end{array}$ \\
\hline 36 & $\begin{array}{c}5.14 \mathrm{E}- \\
09\end{array}$ & $\begin{array}{c}8.31 \mathrm{E}- \\
08\end{array}$ & $\begin{array}{c}2.60 \mathrm{E}- \\
08\end{array}$ & $\begin{array}{c}2.17 \mathrm{E}- \\
08\end{array}$ & $\begin{array}{c}1.44 \mathrm{E}- \\
08\end{array}$ & $\begin{array}{c}8.72 \mathrm{E}- \\
09\end{array}$ & $\begin{array}{c}5.13 \mathrm{E}- \\
09\end{array}$ & $\begin{array}{c}3.29 \mathrm{E}- \\
08\end{array}$ & $\begin{array}{c}2.49 \mathrm{E}- \\
08\end{array}$ & $\begin{array}{c}2.13 \mathrm{E}- \\
08\end{array}$ & $\begin{array}{c}1.44 \mathrm{E}- \\
08\end{array}$ & $\begin{array}{c}8.75 \mathrm{E}- \\
09\end{array}$ & $\begin{array}{c}5.19 \mathrm{E}- \\
09\end{array}$ \\
\hline 37 & $\begin{array}{c}8.20 \mathrm{E}- \\
07\end{array}$ & $\begin{array}{c}8.56 \mathrm{E}- \\
07\end{array}$ & $\begin{array}{c}3.02 \mathrm{E}- \\
08\end{array}$ & $\begin{array}{c}1.14 \mathrm{E}- \\
08\end{array}$ & $\begin{array}{c}9.53 \mathrm{E}- \\
09\end{array}$ & $\begin{array}{c}6.10 \mathrm{E}- \\
09\end{array}$ & $\begin{array}{c}3.75 \mathrm{E}- \\
09\end{array}$ & $\begin{array}{c}8.06 \mathrm{E}- \\
07\end{array}$ & $\begin{array}{c}3.08 \mathrm{E}- \\
08\end{array}$ & $\begin{array}{c}1.11 \mathrm{E}- \\
08\end{array}$ & $\begin{array}{c}9.51 \mathrm{E}- \\
09\end{array}$ & $\begin{array}{c}6.11 \mathrm{E}- \\
09\end{array}$ & $\begin{array}{c}3.72 \mathrm{E}- \\
09\end{array}$ \\
\hline 38 & $\begin{array}{c}2.99 \mathrm{E}- \\
08\end{array}$ & $\begin{array}{c}4.18 \mathrm{E}- \\
08\end{array}$ & $\begin{array}{c}2.53 \mathrm{E}- \\
08\end{array}$ & $\begin{array}{c}2.04 \mathrm{E}- \\
08\end{array}$ & $\begin{array}{c}1.44 \mathrm{E}- \\
08\end{array}$ & $\begin{array}{c}9.83 \mathrm{E}- \\
09\end{array}$ & $\begin{array}{c}\text { 7.14E- } \\
09\end{array}$ & $\begin{array}{c}3.05 \mathrm{E}- \\
08\end{array}$ & $\begin{array}{c}2.51 \mathrm{E}- \\
08\end{array}$ & $\begin{array}{c}2.01 \mathrm{E}- \\
08\end{array}$ & $\begin{array}{c}1.41 \mathrm{E}- \\
08\end{array}$ & $\begin{array}{c}9.86 \mathrm{E}- \\
09\end{array}$ & $\begin{array}{c}7.02 \mathrm{E}- \\
09\end{array}$ \\
\hline 39 & $\begin{array}{c}3.89 \mathrm{E}- \\
08\end{array}$ & $\begin{array}{c}4.02 \mathrm{E}- \\
08\end{array}$ & $\begin{array}{c}3.16 \mathrm{E}- \\
08\end{array}$ & $\begin{array}{c}2.04 \mathrm{E}- \\
08\end{array}$ & $\begin{array}{c}1.25 \mathrm{E}- \\
08\end{array}$ & $\begin{array}{c}7.28 \mathrm{E}- \\
09\end{array}$ & $\begin{array}{c}4.57 \mathrm{E}- \\
09\end{array}$ & $\begin{array}{c}3.91 \mathrm{E}- \\
08\end{array}$ & $\begin{array}{c}3.29 \mathrm{E}- \\
08\end{array}$ & $\begin{array}{c}1.88 \mathrm{E}- \\
08\end{array}$ & $\begin{array}{c}1.12 \mathrm{E}- \\
08\end{array}$ & $\begin{array}{c}7.11 \mathrm{E}- \\
09\end{array}$ & $\begin{array}{c}4.59 \mathrm{E}- \\
09\end{array}$ \\
\hline 40 & $\begin{array}{c}7.03 \mathrm{E}- \\
08\end{array}$ & $\begin{array}{c}8.26 \mathrm{E}- \\
08\end{array}$ & $\begin{array}{c}4.15 \mathrm{E}- \\
08\end{array}$ & $\begin{array}{c}2.71 \mathrm{E}- \\
08\end{array}$ & $\begin{array}{c}2.15 \mathrm{E}- \\
08\end{array}$ & $\begin{array}{c}8.51 \mathrm{E}- \\
09\end{array}$ & $\begin{array}{c}4.54 \mathrm{E}- \\
09\end{array}$ & $\begin{array}{c}5.74 \mathrm{E}- \\
08\end{array}$ & $\begin{array}{c}4.21 \mathrm{E}- \\
08\end{array}$ & $\begin{array}{c}2.78 \mathrm{E}- \\
08\end{array}$ & $\begin{array}{c}2.16 \mathrm{E}- \\
08\end{array}$ & $\begin{array}{c}8.52 \mathrm{E}- \\
09\end{array}$ & $\begin{array}{c}4.51 \mathrm{E}- \\
09\end{array}$ \\
\hline 41 & $\begin{array}{c}2.90 \mathrm{E}- \\
08\end{array}$ & $\begin{array}{c}3.28 \mathrm{E}- \\
08\end{array}$ & $\begin{array}{c}3.15 \mathrm{E}- \\
08\end{array}$ & $\begin{array}{c}2.63 \mathrm{E}- \\
08\end{array}$ & $\begin{array}{c}2.09 \mathrm{E}- \\
08\end{array}$ & $\begin{array}{c}6.44 \mathrm{E}- \\
09\end{array}$ & $\begin{array}{c}4.64 \mathrm{E}- \\
09\end{array}$ & $\begin{array}{c}3.11 \mathrm{E}- \\
08\end{array}$ & $\begin{array}{c}2.75 \mathrm{E}- \\
08\end{array}$ & $\begin{array}{c}2.67 \mathrm{E}- \\
08\end{array}$ & $\begin{array}{c}2.01 \mathrm{E}- \\
08\end{array}$ & $\begin{array}{c}6.45 \mathrm{E}- \\
09\end{array}$ & $\begin{array}{c}3.46 \mathrm{E}- \\
09\end{array}$ \\
\hline 42 & $\begin{array}{c}9.12 \mathrm{E}- \\
08\end{array}$ & $\begin{array}{c}9.10 \mathrm{E}- \\
08\end{array}$ & $\begin{array}{c}5.629 \mathrm{E}- \\
08\end{array}$ & $\begin{array}{c}3.608 \mathrm{E}- \\
08\end{array}$ & $\begin{array}{c}2.42 \mathrm{E}- \\
08\end{array}$ & $\begin{array}{c}1.49 \mathrm{E}- \\
08\end{array}$ & $\begin{array}{c}1.07 \mathrm{E}- \\
08\end{array}$ & $\begin{array}{c}9.18 \mathrm{E}- \\
08\end{array}$ & $\begin{array}{c}5.93 \mathrm{E}- \\
08\end{array}$ & $\begin{array}{c}3.428 \mathrm{E}- \\
08\end{array}$ & $\begin{array}{c}2.552 \mathrm{E}- \\
08\end{array}$ & $\begin{array}{c}1.50 \mathrm{E}- \\
08\end{array}$ & $\begin{array}{c}1.05 \mathrm{E}- \\
08\end{array}$ \\
\hline 43 & $\begin{array}{c}9.46 \mathrm{E}- \\
07\end{array}$ & $\begin{array}{c}9.00 \mathrm{E}- \\
07\end{array}$ & $\begin{array}{c}3.14 \mathrm{E}- \\
08\end{array}$ & $\begin{array}{c}2.212 \mathrm{E}- \\
08\end{array}$ & $\begin{array}{c}1.9 \mathrm{E}- \\
08\end{array}$ & $\begin{array}{c}1.13 \mathrm{E}- \\
08\end{array}$ & $\begin{array}{c}7.18 \mathrm{E}- \\
09\end{array}$ & $\begin{array}{c}9.40 \mathrm{E}- \\
07\end{array}$ & $\begin{array}{c}2.92 \mathrm{E}- \\
08\end{array}$ & $\begin{array}{c}2.348 \mathrm{E}- \\
08\end{array}$ & $1.9 \mathrm{E}-08$ & $\begin{array}{c}1.02 \mathrm{E}- \\
08\end{array}$ & $\begin{array}{c}7.13 \mathrm{E}- \\
09\end{array}$ \\
\hline 44 & $\begin{array}{c}1.65 \mathrm{E}- \\
08\end{array}$ & $\begin{array}{c}2.59 \mathrm{E}- \\
08\end{array}$ & $\begin{array}{c}2.11 \mathrm{E}- \\
08\end{array}$ & $\begin{array}{c}1.194 \mathrm{E}- \\
08\end{array}$ & $\begin{array}{c}6.83 \mathrm{E}- \\
09\end{array}$ & $\begin{array}{c}4.96 \mathrm{E}- \\
09\end{array}$ & $\begin{array}{c}3.93 \mathrm{E}- \\
09\end{array}$ & $\begin{array}{c}2.00 \mathrm{E}- \\
08\end{array}$ & $\begin{array}{c}1.66 \mathrm{E}- \\
08\end{array}$ & $\begin{array}{c}1.136 \mathrm{E}- \\
08\end{array}$ & $\begin{array}{c}6.93 \mathrm{E}- \\
09\end{array}$ & $\begin{array}{c}4.80 \mathrm{E}- \\
09\end{array}$ & $\begin{array}{c}3.97 \mathrm{E}- \\
09\end{array}$ \\
\hline 45 & $\begin{array}{c}1.55 \mathrm{E}- \\
07\end{array}$ & $\begin{array}{c}1.77 \mathrm{E}- \\
07\end{array}$ & $\begin{array}{c}4.87 \mathrm{E}- \\
08\end{array}$ & $\begin{array}{c}2.33 \mathrm{E}- \\
08\end{array}$ & $\begin{array}{c}7.3 \mathrm{E}- \\
09\end{array}$ & $\begin{array}{c}6.40 \mathrm{E}- \\
09\end{array}$ & $\begin{array}{c}4.11 \mathrm{E}- \\
09\end{array}$ & $\begin{array}{c}1.46 \mathrm{E}- \\
07\end{array}$ & $\begin{array}{c}6.35 \mathrm{E}- \\
08\end{array}$ & $\begin{array}{c}2.24 \mathrm{E}- \\
08\end{array}$ & $\begin{array}{c}7.22 \mathrm{E}- \\
09\end{array}$ & $\begin{array}{c}6.82 \mathrm{E}- \\
09\end{array}$ & $\begin{array}{c}4.16 \mathrm{E}- \\
09\end{array}$ \\
\hline 46 & $\begin{array}{c}1.91 \mathrm{E}- \\
06\end{array}$ & $\begin{array}{c}8.31 \mathrm{E}- \\
06\end{array}$ & $\begin{array}{c}7.67 \mathrm{E}- \\
08\end{array}$ & $\begin{array}{c}6.79 \mathrm{E}- \\
08\end{array}$ & $\begin{array}{c}2.02 \mathrm{E}- \\
08\end{array}$ & $\begin{array}{c}1.58 \mathrm{E}- \\
08\end{array}$ & $\begin{array}{c}8.69 \mathrm{E}- \\
09\end{array}$ & $\begin{array}{c}4.13 \mathrm{E}- \\
06\end{array}$ & $\begin{array}{c}5.74 \mathrm{E}- \\
08\end{array}$ & $\begin{array}{c}3.08 \mathrm{E}- \\
08\end{array}$ & $\begin{array}{c}2.09 \mathrm{E}- \\
08\end{array}$ & $\begin{array}{c}1.56 \mathrm{E}- \\
08\end{array}$ & $\begin{array}{c}8.76 \mathrm{E}- \\
09\end{array}$ \\
\hline 47 & $\begin{array}{c}1.67 \mathrm{E}- \\
07\end{array}$ & $\begin{array}{c}1.77 \mathrm{E}- \\
07\end{array}$ & $\begin{array}{c}2.87 \mathrm{E}- \\
08\end{array}$ & $\begin{array}{c}2.25 \mathrm{E}- \\
08\end{array}$ & $\begin{array}{c}2.18 \mathrm{E}- \\
08\end{array}$ & $\begin{array}{c}1.79 \mathrm{E}- \\
08\end{array}$ & $\begin{array}{c}1.33 \mathrm{E}- \\
08\end{array}$ & $\begin{array}{c}1.70 \mathrm{E}- \\
07\end{array}$ & $\begin{array}{c}2.84 \mathrm{E}- \\
08\end{array}$ & $\begin{array}{c}2.26 \mathrm{E}- \\
08\end{array}$ & $\begin{array}{c}2.12 \mathrm{E}- \\
08\end{array}$ & $\begin{array}{c}1.71 \mathrm{E}- \\
08\end{array}$ & $\begin{array}{c}1.35 \mathrm{E}- \\
08\end{array}$ \\
\hline 48 & $\begin{array}{c}6.23 \mathrm{E}- \\
05\end{array}$ & $\begin{array}{c}5.40 \mathrm{E}- \\
05\end{array}$ & $\begin{array}{c}3.26 \mathrm{E}- \\
08\end{array}$ & $\begin{array}{c}2.20 \mathrm{E}- \\
08\end{array}$ & $\begin{array}{c}1.54 \mathrm{E}- \\
08\end{array}$ & $\begin{array}{c}1.29 \mathrm{E}- \\
08\end{array}$ & $\begin{array}{c}8.06 \mathrm{E}- \\
09\end{array}$ & $\begin{array}{c}5.43 \mathrm{E}- \\
05\end{array}$ & $\begin{array}{c}3.40 \mathrm{E}- \\
08\end{array}$ & $\begin{array}{c}2.25 \mathrm{E}- \\
08\end{array}$ & $\begin{array}{c}1.56 \mathrm{E}- \\
08\end{array}$ & $\begin{array}{c}1.27 \mathrm{E}- \\
08\end{array}$ & $\begin{array}{c}8.03 \mathrm{E}- \\
09\end{array}$ \\
\hline 49 & $\begin{array}{c}2.37 \mathrm{E}- \\
07\end{array}$ & $\begin{array}{c}2.46 \mathrm{E}- \\
07\end{array}$ & $\begin{array}{c}3.23 \mathrm{E}- \\
08\end{array}$ & $\begin{array}{c}2.92 \mathrm{E}- \\
08\end{array}$ & $\begin{array}{c}2.03 \mathrm{E}- \\
08\end{array}$ & $\begin{array}{c}1.16 \mathrm{E}- \\
08\end{array}$ & $\begin{array}{c}6.21 \mathrm{E}- \\
09\end{array}$ & $\begin{array}{c}2.55 \mathrm{E}- \\
07\end{array}$ & $\begin{array}{c}3.05 \mathrm{E}- \\
08\end{array}$ & $\begin{array}{c}2.94 \mathrm{E}- \\
08\end{array}$ & $\begin{array}{c}2.04 \mathrm{E}- \\
08\end{array}$ & $\begin{array}{c}1.15 \mathrm{E}- \\
08\end{array}$ & $\begin{array}{c}6.10 \mathrm{E}- \\
09\end{array}$ \\
\hline 50 & $\begin{array}{c}1.34 \mathrm{E}- \\
07\end{array}$ & $\begin{array}{c}1.57 \mathrm{E}- \\
07\end{array}$ & $\begin{array}{c}3.92 \mathrm{E}- \\
08\end{array}$ & $\begin{array}{c}1.66 \mathrm{E}- \\
08\end{array}$ & $\begin{array}{c}1.45 \mathrm{E}- \\
08\end{array}$ & $\begin{array}{c}9.45 \mathrm{E}- \\
09\end{array}$ & $\begin{array}{c}7.79 \mathrm{E}- \\
09\end{array}$ & $\begin{array}{c}1.53 \mathrm{E}- \\
07\end{array}$ & $\begin{array}{c}3.91 \mathrm{E}- \\
08\end{array}$ & $\begin{array}{c}1.61 \mathrm{E}- \\
08\end{array}$ & $\begin{array}{c}1.42 \mathrm{E}- \\
08\end{array}$ & $\begin{array}{c}9.63 \mathrm{E}- \\
09\end{array}$ & $\begin{array}{c}7.80 \mathrm{E}- \\
09\end{array}$ \\
\hline 51 & $\begin{array}{c}1.63 \mathrm{E}- \\
08\end{array}$ & $\begin{array}{c}1.89 \mathrm{E}- \\
08\end{array}$ & $\begin{array}{c}1.25 \mathrm{E}- \\
08\end{array}$ & $\begin{array}{c}1.21 \mathrm{E}- \\
08\end{array}$ & $\begin{array}{c}1.12 \mathrm{E}- \\
08\end{array}$ & $\begin{array}{c}5.78 \mathrm{E}- \\
09\end{array}$ & $\begin{array}{c}3.93 \mathrm{E}- \\
09\end{array}$ & $\begin{array}{c}1.82 \mathrm{E}- \\
08\end{array}$ & $\begin{array}{c}1.501 \mathrm{E}- \\
08\end{array}$ & $\begin{array}{c}1.31 \mathrm{E}- \\
08\end{array}$ & $\begin{array}{c}1.14 \mathrm{E}- \\
08\end{array}$ & $\begin{array}{c}5.70 \mathrm{E}- \\
09\end{array}$ & $\begin{array}{c}3.97 \mathrm{E}- \\
09\end{array}$ \\
\hline 52 & $\begin{array}{c}1.52 \mathrm{E}- \\
08\end{array}$ & $\begin{array}{c}4.48 \mathrm{E}- \\
08\end{array}$ & $\begin{array}{c}4.27 \mathrm{E}- \\
08\end{array}$ & $\begin{array}{c}2.54 \mathrm{E}- \\
08\end{array}$ & $\begin{array}{c}1.98 \mathrm{E}- \\
08\end{array}$ & $\begin{array}{c}1.86 \mathrm{E}- \\
08\end{array}$ & $\begin{array}{c}3.01 \mathrm{E}- \\
09\end{array}$ & $\begin{array}{c}3.36 \mathrm{E}- \\
08\end{array}$ & $\begin{array}{c}4.31 \mathrm{E}- \\
08\end{array}$ & $\begin{array}{c}2.95 \mathrm{E}- \\
08\end{array}$ & $\begin{array}{c}2.13 \mathrm{E}- \\
08\end{array}$ & $\begin{array}{c}2.01 \mathrm{E}- \\
08\end{array}$ & $\begin{array}{c}2.86 \mathrm{E}- \\
09\end{array}$ \\
\hline 53 & $\begin{array}{c}5.00 \mathrm{E}- \\
07\end{array}$ & $\begin{array}{c}5.79 \mathrm{E}- \\
07\end{array}$ & $\begin{array}{c}2.25 \mathrm{E}- \\
08\end{array}$ & $\begin{array}{c}7.99 \mathrm{E}- \\
09\end{array}$ & $\begin{array}{c}5.09 \mathrm{E}- \\
09\end{array}$ & $\begin{array}{c}4.77 \mathrm{E}- \\
09\end{array}$ & $\begin{array}{c}3.99 \mathrm{E}- \\
09\end{array}$ & $\begin{array}{c}4.10 \mathrm{E}- \\
07\end{array}$ & $\begin{array}{c}6.87 \mathrm{E}- \\
08\end{array}$ & $5.2 \mathrm{E}-09$ & $\begin{array}{c}5.02 \mathrm{E}- \\
09\end{array}$ & $\begin{array}{c}3.05 \mathrm{E}- \\
09\end{array}$ & $\begin{array}{c}2.55 \mathrm{E}- \\
09\end{array}$ \\
\hline 54 & $\begin{array}{c}4.59 \mathrm{E}- \\
06\end{array}$ & $\begin{array}{c}4.59 \mathrm{E}- \\
06\end{array}$ & $\begin{array}{c}1.93 \mathrm{E}- \\
08\end{array}$ & $\begin{array}{c}8.76 \mathrm{E}- \\
09\end{array}$ & $\begin{array}{c}6.58 \mathrm{E}- \\
09\end{array}$ & $\begin{array}{c}\text { 4.79E- } \\
09\end{array}$ & $\begin{array}{c}4.65 \mathrm{E}- \\
09\end{array}$ & $\begin{array}{c}4.60 \mathrm{E}- \\
06\end{array}$ & $\begin{array}{c}2.54 \mathrm{E}- \\
08\end{array}$ & $\begin{array}{c}8.64 \mathrm{E}- \\
09\end{array}$ & $\begin{array}{c}6.54 \mathrm{E}- \\
09\end{array}$ & $\begin{array}{c}4.82 \mathrm{E}- \\
09\end{array}$ & $\begin{array}{c}4.76 \mathrm{E}- \\
09\end{array}$ \\
\hline
\end{tabular}


The relation between loading time and permeability for each loading stage drown this permeability found by specifying the degree of consolidation as mention before, this relation is shown in figure (2) for sample number 52

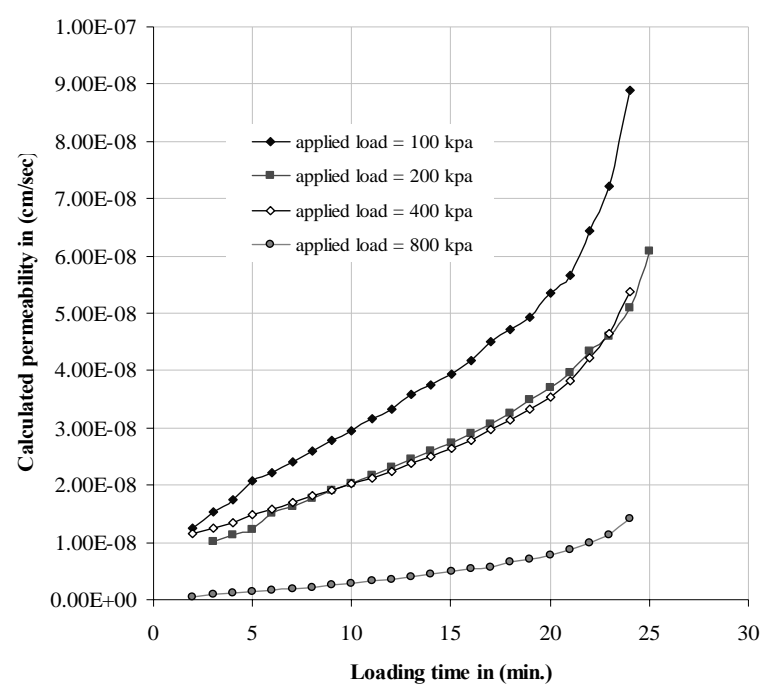

Figure (2): relation between loading time and calculated permeability for different applied load (for sample no. 52)

From this figure it found that the permeability changed with time that mean that the calculated permeability not accurate for any degree of consolidation., therefore this relation reputed for all the samples which is number is 54 sample and the coefficient of permeability calculated for each minute $(1,2,3 \ldots 24)$ minuet and this results studied and analysis made to find the suitable time that could be used to give accurate permeability and this time found at (10 minute) and each loading stage lasted at least for (25 minute)

\section{Consolidation ratio and permeability coefficient:}

From the experiment that made for this research it found that the consolidation ratio for almost cases greater than 60 \%Therefore equation 18 simplified to became as: 


$$
\begin{aligned}
k & =\frac{T}{1200000001 P}\left[\left(1.9-0.0002\left(R_{0(p=0)}-R_{\text {final }(P=-i-1)}\right)\right)-\left(0.0001\left(R_{10(P=i)}-R_{0(P=i)}\right)\right)\right]^{2} \\
& \times\left[\frac{\left(R_{10(P-i)}-R_{0(P=i)}\right)}{\left(1.9-0.0002\left(R_{0(p=0)}-R_{\text {final }(P-i-1)}\right)\right)}\right]
\end{aligned}
$$

Where:

$$
T=1.781-0.933 \log \left(100\left(1-\frac{\left(R_{10(P=i)}-R_{O(P=i)}\right)}{\left(R_{25(P=i)}-R_{O(P=i)}\right)}\right)\right)
$$

$R_{0(p=0)}=$ dial reading at the start of testing, time $=0$ minute at applied load $=0$ $\mathrm{kpa}$

$R_{0(P=i-1)}=$ dial reading at the start of previous loading stage.

$R_{10(p=i)}=$ dial reading at the proposed time which equal to ten minute after starting applying the load.

$R_{25 \text { ( } p=i)}=$ dial reading at the end of applying the load which equal to 25 minute after starting applying the load.

The results studied to find the compatibility of the proposed procedure using derived equation (23) and the ordinary direct method (falling head method) as shown in figure (3) bellow: 


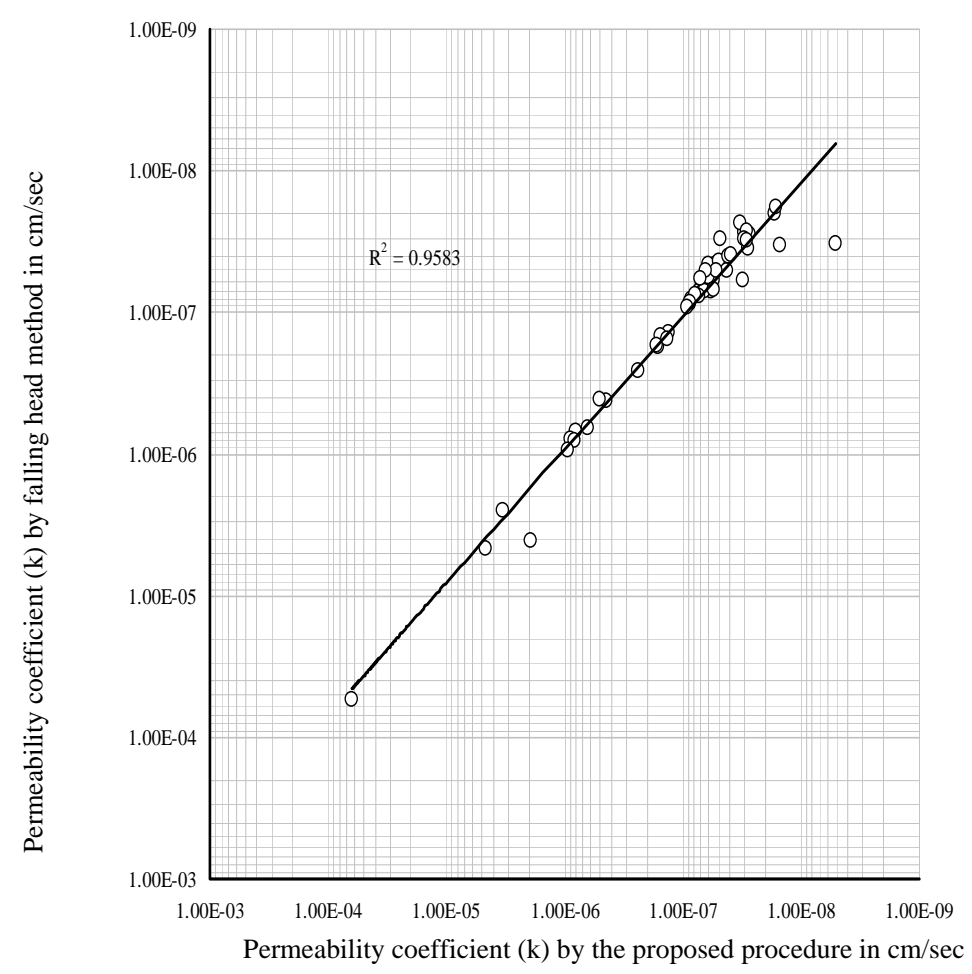

Figure (3): relation of permeability coefficient $(k)$ between the proposed procedure and falling head method from my experimental results.

As shown in figure (3) the proposed procedure give a very good compatibility with the direct method ( falling head method) with root square equal to (0.9583) and correlation factor equal to (0.9988).

\section{Verification of the proposed procedure:}

\section{Case study number1:}

In 1980 Bowles published ordinary consolidation test results of soil taken from Bradley University in his book "engineering properties of soils and their measurement”. This data used to find the permeability indirectly (using the consolidation test) for applied load equal to 100 and $200(\mathrm{kPa})$ this result compare with the proposed equation as shown in table (4) and give correlation factor almost to (0.999999). 
Table (4) case study no. 1

\begin{tabular}{|c|c|c|}
\hline \multirow{2}{*}{$\begin{array}{c}\text { Applied load } \\
(\mathrm{kPa})\end{array}$} & \multicolumn{2}{|c|}{ permeability coefficient (k) (cm/sec) } \\
\cline { 2 - 3 } & Ordinary Consolidation Method & Proposed Procedure \\
\hline 100 & 3.61 E-10 & 6.14 E-10 \\
\hline 200 & 3.55 E-10 & 4.74 E-10 \\
\hline
\end{tabular}

\section{Case study number 2:}

In 1963 Taylor published ordinary consolidation test results of Chicago clay sample in his book "fundamentals of soil mechanics". This data used to find the permeability indirectly (using the consolidation test) for applied load equal to $(25,50,100,200,400,800$ and 1600) (kPa) this result compare with the proposed procedure using derive equation as shown in figure (4) and give correlation factor equal to ( 0.989413$)$

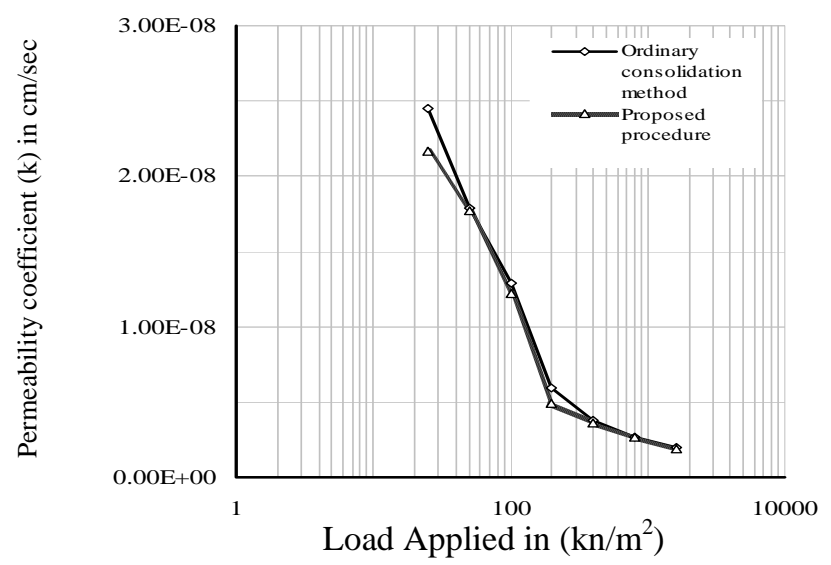

Figure (4): relation between the permeability coefficient (k) and the applied load for the ordinary consolidation method and the proposed procedure. 


\section{Conclusions:}

The following conclusions can be drawn from the results of this research:

1- It is found that the proposed equation which is depending on the rate of consolidation give very accurate results to fined the permeability of soils.

2- This proposed equation could be accurate with clayey soils.

3- The proposed procedure was very fast and gives a very good results comparing with ordinary method to found the permeability especially with clayey soils.

4- The proposed equation give a good compatibility with the direct method ( falling head method) where the root square equal was (0.9583) and correlation factor equal to (0.9988)

\section{List of Abbreviations}

$D_{10}=$ the effective size in $\mathrm{mm}$

$C=$ a coefficient varying between 0.01 and 0.015

$k_{o}$ is a factor depending on the shape of the pores.

$k_{T}$ is a factor depending on the 'tortuosity' of the pores (that is , no the mean distance traveled by the water in moving between two points which are unit distance apart in a straight line).

$S_{s}$ is the surface area of the particles in unit volume of the solid material.

$\gamma_{w}$ is the unit weight of permeant

$\mu=$ viscosity of permeant

$n=$ porosity

$e_{0}=$ initial void ratio.

$e_{i}=$ the void ratio at the load increment

$\Delta e=$ corresponding change in void ratio.

$\Delta p=$ change in applied stress

$k=$ coefficient of permeability.

$m_{v}=$ coefficient of volume change.

$a_{v}=$ coefficient of compressibility

$C_{v}=$ coefficient of consolidation.

$H_{a v .}=$ average height of sample 
$T$ = time factor

$t_{T}=$ time for the corresponding time factor $T$

$\varepsilon_{v}=$ volumetric strain.

$\varepsilon_{r}=$ Radial strain

$\varepsilon_{h}=$ axial strain.

$H_{O(P=i)}=$ initial height at that loading stage.

$H_{t(P=i)}=$ height of sample at the proposed reading time.

$H_{\text {final }(P=i)}=$ height of sample at the end of this loading stage.

$R_{O(P=i)}=$ dial gage reading at the start of that loading stage.

$R_{t(P=i)}=$ dial gage reading at the proposed reading time of that loading stage.

$R_{\text {final }(P=i)}=$ dial gage reading at the end of this loading stage.

$\mathrm{H}_{0(\mathrm{p}=0)}=$ initial height of the sample at applied load $=0 \mathrm{kpa}$

$R_{0(p=0)}=$ dial reading at the start of testing, time $=0$ min at applied load $=0$ $\mathrm{kpa}$

$R_{\text {final( } P=i-1)}=$ dial reading at the end of previous loading stage.

$R_{0(p=0)}=$ dial reading at the start of testing, time $=0$ minute at applied load $=0$ kpa

$R_{0(P=i-1)}=$ dial reading at the start of previous loading stage.

$R_{10(p=i)}=$ dial reading at the proposed time which equal to ten minute after starting applying the load.

$R_{25(p=i)}=$ dial reading at the end of applying the load which equal to 25 minute after starting applying the load.

$i=$ hydraulic gradient $h / L$

$L=$ flow path

$h=$ hydraulic head

$Q=$ The quantity of flow

$\mathrm{A}=\mathrm{a}$ cross section of area 
$v=$ discharge velocity, which is due quantity of water flowing in unit time through a unit gross cross section area of soil at right angle to the direction of flow.

\section{References:}

[1] Bowles, J.E. 1981,"Engineering properties of soil and their measurement", McGraw-HILL Book Company, inc. (second edition).

[2] Carman, P.C.1956, "Flow of Gases through Porous Media" .Academic Press (New York).

[3] Darcy ,H. 1856 ,"les fountains publiques de la ville de Dijon". Paris: Dijon.

[4] Gregory, P.T.1955," Soil mechanics, Foundations, and earth structures". McGraw-HILL Book Company, inc. (New York).

[5] Holtz R.D. and Kovacs W.D., 1981,"An introduction to geotechnical engineering". Prentice-hall (New Jersey).pp. (383-385).

[6] Scott, C.R. 1980, "Soil mechanics and foundations ".Applied Science Publishers (London).

[7] Taylor, D. W. 1963, "Soil mechanics". John Wiley and Sons (New York). 